NBER WORKING PAPER SERIES

\author{
RATIONALLY INATTENTIVE BEHAVIOR: \\ CHARACTERIZING AND GENERALIZING SHANNON ENTROPY \\ Andrew Caplin \\ Mark Dean \\ John Leahy \\ Working Paper 23652 \\ http://www.nber.org/papers/w23652
}

NATIONAL BUREAU OF ECONOMIC RESEARCH

1050 Massachusetts Avenue

Cambridge, MA 02138

August 2017

We thank Dirk Bergemann, Daniel Csaba, Henrique de Oliveira, Xavier Gabaix, Sen Geng, Andrei Gomberg, Michael Magill, Daniel Martin, Filip Matejka, Alisdair McKay, Stephen Morris, Efe Ok, and Michael Woodford for their constructive contributions. This paper builds on the material contained in the working paper "The Behavioral Implications of Rational Inattention with Shannon Entropy" by Andrew Caplin and Mark Dean [2013], and subsumes all common parts. The views expressed herein are those of the authors and do not necessarily reflect the views of the National Bureau of Economic Research.

NBER working papers are circulated for discussion and comment purposes. They have not been peer-reviewed or been subject to the review by the NBER Board of Directors that accompanies official NBER publications.

(C) 2017 by Andrew Caplin, Mark Dean, and John Leahy. All rights reserved. Short sections of text, not to exceed two paragraphs, may be quoted without explicit permission provided that full credit, including $\odot$ notice, is given to the source. 
Rationally Inattentive Behavior: Characterizing and Generalizing Shannon Entropy Andrew Caplin, Mark Dean, and John Leahy

NBER Working Paper No. 23652

August 2017

JEL No. D8,D83

\begin{abstract}
$\underline{\text { ABSTRACT }}$
We provide a full behavioral characterization of the standard Shannon model of rational inattention. The key axiom is "Invariance under Compression", which identifies this model as capturing an ideal form of attention-constrained choice. We introduce tractable generalizations that allow for many of the known behavioral violations from this ideal, including asymmetries and complementarities in learning, context effects, and low responsiveness to incentives. We provide an even more general method of recovering attention costs from behavioral data. The data set in which we characterize all behavioral patterns is "state dependent" stochastic choice data.

Andrew Caplin

Department of Economics

New York University

19 W. 4th Street, 6th Floor

New York, NY 10012

and NBER

andrew.caplin@nyu.edu

Mark Dean

Columbia University

$420 \mathrm{~W} 118$ th Street

New York, NY 10027

Mark.Dean@columbia.edu

John Leahy

Gerald R. Ford School of Public Policy

University of Michigan

3308 Weill Hall

735 S. State St. \#3308

Ann Arbor, MI 48109

and NBER

jvleahy@umich.edu
\end{abstract}

An online appendix is available at http://www.nber.org/data-appendix/w23652 


\title{
Rationally Inattentive Behavior: Characterizing and Generalizing Shannon Entropy*
}

\author{
Andrew Caplin† Mark Dean ${ }^{\ddagger}$ and John Leahy ${ }^{\S}$
}

July 2017

\begin{abstract}
We provide a full behavioral characterization of the standard Shannon model of rational inattention. The key axiom is "Invariance under Compression", which identifies this model as capturing an ideal form of attention-constrained choice. We introduce tractable generalizations that allow for many of the known behavioral violations from this ideal, including asymmetries and complementarities in learning, context effects, and low responsiveness to incentives. We provide an even more general method of recovering attention costs from behavioral data. The data set in which we characterize all behavioral patterns is "state dependent" stochastic choice data.
\end{abstract}

\section{Introduction}

Understanding limits on private information has been central to economic analysis since the pioneering work of Hayek [1937, 1945]. While there are many models of information acquisition in use (see Hellwig et al. [2012]), a major new route to such understanding was initiated by Sims $[1998,2003]$, who introduced the theory of rational inattention. He considered the implications of attention costs based on Shannon mutual information for macroeconomic dynamics. The ensuing period has seen applications of the Shannon cost function to such diverse subjects as stochastic choice (Matejka and McKay [2015]), investment decisions (Mondria [2010]), global games (Yang [2015]), pricing decisions (Woodford [2009], Mackowiak and Wiederholt [2009], Martin [2017] and Matějka [2015]), dynamic learning (Steiner et al. [2015]) and social learning (Caplin et al. [2015]).

One reason for the appeal of the Shannon cost function is analytic tractability. A second lies is its connection to optimal coding (Shannon [1948], Sims [2003], Cover and Thomas [2012]). Yet

*We thank Dirk Bergemann, Daniel Csaba, Henrique de Oliveira, Xavier Gabaix, Sen Geng, Andrei Gomberg, Michael Magill, Daniel Martin, Filip Matejka, Alisdair McKay, Stephen Morris, Efe Ok, and Michael Woodford for their constructive contributions. This paper builds on the material contained in the working paper "The Behavioral Implications of Rational Inattention with Shannon Entropy" by Andrew Caplin and Mark Dean [2013], and subsumes all common parts.

${ }^{\dagger}$ Center for Experimental Social Science and Department of Economics, New York University. Email: andrew.caplin@nyu.edu

${ }^{\ddagger}$ Department of Economics, Columbia University. Email: mark.dean@columbia.edu

${ }^{\S}$ Department of Economics, Universtity of Michigan and NBER. Email: jvleahy@umich.edu 
these do not imply behavioral validity. Indeed it is now known that behavior often violates key features of the Shannon model. These include: its implication that all states are equally easy to identify and to discriminate among (see Dewan and Neligh [2017] for behavioral counterexamples); the implied flexibility of response to payoff incentives (see Caplin and Dean [2013] for behavioral counterexamples); and essential independence of behavior from event likelihoods (see Woodford [2012] for behavioral counter examples).

At this stage there are two related open questions. First, while various features of the behavior associated with the Shannon model are known, there is as yet no full behavioral characterization. What precisely are the behavioral characteristics of the Shannon model? Second, what workable alternative models allow for the complex behavioral patterns identified in practice?

In this paper we address both questions. We provide a full behavioral characterization of the Shannon model. This characterization pinpoints it as defining an "ideal" form of attentionconstrained choice, in which choices depend only on the probabilistic structure of payoffs. We also introduce two tractable generalizations that allow for many observed behavioral departures from the Shannon model. The first involves Uniformly Posterior-Separable (UPS) models, in which attention costs depend on the expectation of a general convex function of the posterior beliefs. One particular example uses Tsallis entropy instead of Shannon entropy. UPS models allow for such factors as: asymmetric costs of learning about distinct states; differing perceptual distance between distinct states; complementarities in learning about distinct states; and complex responses to payoff incentives. Posterior-separable (PS) models are even more flexible as they allow the cost of attention to depend on prior beliefs and hence vary from context to context. The tractability of PS and UPS models derives from the fact that, as in the Shannon model, attention costs depend on the expectation of a strictly convex function of posterior beliefs so that standard Lagrangian optimization methods are available. These generalizations are already gaining traction in the literature (see Caplin and Dean [2013], Gentzkow and Kamenica [2014], Steiner et al. [2015], Clark [2016] and Morris and Strack [2017]).

In addition to answering open questions, our analysis produces an unexpected bonus. We provide a constructive method of recovering attention costs from behavioral data. This method is both general and intuitively reasonable, resting as it does on standard balancing of marginal costs and marginal utility.

Our first result starts from behavioral patterns induced by a general UPS cost function and identifies additional restrictions that ensure that this function is of the Shannon form. UPS cost functions are characterized by a general strictly convex function of posterior beliefs. The Shannon model specializes to a particular one parameter family of such functions. Hence the gap between the UPS model and the Shannon model can be analogized to that between a general strictly concave utility function and Cobb-Douglas utility.

Just as constancy of expenditure shares pins down the Cobb-Douglas form, we identify a single behavioral axiom that defines the Shannon model. Invariance Under Compression (IUC) makes choices invariant to changes in the underlying state space that do not impact the probabilistic structure of payoffs. In this sense, the Shannon model alone produces an idealized form of attentionconstrained behavior in which only payoff relevant information matters.

Our remaining results establish necessary and sufficient conditions for a UPS representation. They do this in three stages, with each stage being of independent interest. The first stage introduces axioms for recoverability of the cost function. The second introduces additional axioms and 
characterizes the PS model. The third adds one final axiom that specializes the PS to the UPS model.

With regard to recoverability, we show that the cost function is fully pinned down by three behavioral axioms. The first two are necessary for existence of a rationalizing cost function of any form: no improving action switches (NIAS: see Caplin and Martin [2015]) and no improving attention cycles (NIAC; see Caplin and Dean [2015]). The final axiom requires "completeness" of the behavioral data, in a sense to be made precise.

The PS characterization relies mainly on a behavioral invariance axiom, Separability, that is less restrictive than IUC. It enables one to replace any action in a given decision problem without disturbing the behavior associated with common actions. There are also three axioms that address technical issues in the representation.

The UPS characterization rests on a third behavioral invariance property, Locally Invariant Posteriors (LIP), intermediate in generality between Separability and IUC. LIP insists that behavioral data derived from any given decision problem can be used to characterize data for a wide class of related problems, subject to Bayesian consistency.

Central to our approach is a particular specification of the choice data available to an ideal observer, such as an econometrician or economic theorist. One key feature is stochasticity. That cognitive constraints produce stochasticity in choice has been a commonplace in psychometrics ever since the pioneering perceptual experiments of Weber [1834] and the formal models of Thurstone [1927] and Luce [1959]. Stochasticity is also a feature in all models of rational inattention which focus on cognitively constrained updating. Yet standard stochastic choice data is fundamentally inadequate to capture attention, since it does not measure the degree of match between behavior and reality. As Block and Marschak [1960] (p. 98-99) presciently noted, the path forward in testing theories of attention lies in data enrichment.

The data set that we study is "state-dependent" stochastic choice (SDSC) data, as introduced in Caplin and Martin [2015] and Caplin and Dean [2015]. This treats both the payoff determining states of the world and the behavioral choice as observable. It rests on the idea that attentional constraints do not apply to an ideal observer. While consumers may have difficulty assessing whether or not sales tax is included in the price paid at the register, the econometrician knows (Chetty et al. [2009]). The resulting data strongly reflects the match between perception and reality. In fact our results show that SDSC data can capture the full behavioral footprint of attention costs, in stark contrast with standard stochastic choice data.

Our work is related to a growing recent literature aimed at understanding the behavioral implications of models with limited attention. Notable recent contributions include Masatlioglu et al. [2012], Manzini and Mariotti [2014], Oliveira et al. [2017] and Steiner and Stewart [2016]. More specifically, there have been several recent papers which have used first order conditions to solve the Shannon model (for example Stevens [2014], Matejka and McKay [2015], Steiner et al. [2015], Caplin et al. [2016]). Unlike these papers, we provide a set of easily interpretable axioms which give insight into the type of behavioral patterns that the Shannon model predicts. de Oliveira [2013] considers the behavioral implications of the Shannon model, but for a data set which consists of observed choices over different menus of alternatives. Instead, our work uses SDSC, and therefore links in with the recent renewed interest in modelling random choice in general (e.g. Agranov and Ortoleva [2015], Manzini and Mariotti [2016], Apesteguia and Ballester [2016]), and it's relationship to information acquisition in particular (e.g. Krajbich and Rangel [2011]). 
Section 2 defines attention strategies in analytically appropriate form, and introduces the various classes of attention cost functions. Section 3 establishes general applicability of Lagrangian methods of identifying optimal strategies. Section 4 introduces SDSC data and links it to attention strategies. Section 5 introduces our IUC axiom and the associated characterization theorem. Section 6 introduces the recoverability result. Our characterizations of the PS and UPS models are in Section 7. Section 8 provides additional analyses concerning alternative formulations of the representation theorems and the properties of our axioms. Section 9 relates our work to the existing literature on attention. Section 10 concludes. Throughout the paper we present the main Theorems and discuss informally why they are true. Formal proofs are in the Appendix.

\section{Attention Strategies and Costs}

\subsection{Posterior-Based Attention Strategies}

We consider a decision maker (DM) who faces a large class of decision problems related to an infinite (countable or uncountable) underlying set $\Omega$ of conceivable states of the world and an uncountably infinite set of potentially available actions, $\mathcal{A}$. In a given decision problem, the DM is endowed with a prior with finite support as well as a finite set of available actions. The DM receives known expected utility $u(a, \omega)$ when action $a \in \mathcal{A}$ is chosen in state $\omega \in \Omega$. We assume that $\mathcal{A}$ is rich enough that values $u(a, \omega) \in \mathbb{R}$ are unrestricted.

Definition 1 Given $\mu \in \Delta(\Omega) \equiv \Gamma, \Omega(\mu) \equiv\{\omega \in \Omega \mid \mu(\omega)>0\}$ specifies possible states (where $\Delta$ denote simple distributions over the space); $\Gamma(\mu)=\{\gamma \in \Gamma \mid \Omega(\gamma) \subset \Omega(\mu)\}$ possible posteriors; and $\tilde{\Gamma}(\mu)=\{\gamma \in \Gamma(\mu) \mid \Omega(\gamma)=\Omega(\mu)\}$ interior posteriors with precisely the same support as $\mu$.

Definition 2 A decision problem comprises a pair $(\mu, A) \in \Gamma \times \mathcal{A}$ with $A$ finite. $\mathcal{D}$ is the set of such decision problems.

The central decision that we model concerns how much to learn. The DM decides this by comparing the incremental improvement in decision quality associated with improved information with the cost of incremental information. In formalizing the cost of learning, we will focus on the outcome of the learning process and assign costs directly to each Bayes-consistent distribution of posteriors, as in Caplin and Dean (2013). To this end, we define an attention strategy in terms of the resulting posteriors and their implications for choice.

Definition 3 Given $(\mu, A) \in \mathcal{D}$, the set of posterior-based attention strategies comprises all simple probability distributions over posteriors and corresponding mixed action strategies,

$$
\Lambda(\mu, A) \equiv\left\{\lambda=\left(Q_{\lambda}, q_{\lambda}\right) \mid Q_{\lambda} \in \mathcal{Q}(\mu), q_{\lambda}: \Gamma\left(Q_{\lambda}\right) \rightarrow \Delta(A)\right\}
$$

with $\mathcal{A}(\lambda) \subset A$ the chosen actions, and $\mathcal{Q}(\mu)$ the Bayes-consistent distributions,

$$
\mathcal{Q}(\mu)=\left\{Q \in \Delta(\Gamma(\mu)) \mid \mu=\sum_{\gamma \in \Gamma(Q)} \gamma Q(\gamma)\right\}
$$


We define corresponding unions $\Lambda(\mu)$ and $\Lambda$, and also define $\Lambda^{I}(\mu) \subset \Lambda(\mu)$ as the set of inattentive strategies such that $\Gamma\left(Q_{\lambda}\right)=\mu$.

This posterior-based approach departs from the standard signal-based approach which specifies the cost of an available set of signals correlated with the true state of the world (see for example Caplin and Dean [2015]). There are two key advantages of the posterior-based formulation. First, our behavioral characterizations are more naturally stated in terms of posteriors. Second, this formulation allows for several interesting generalizations of the Shannon cost function. Of course, there is in general a mapping between signals and posteriors. We discuss in Section 8 why behavioral results are independent of how strategies are formulated.

Figure 1 illustrates the strategy $\lambda^{*}$ which we use as a running example. The underlying decision problem consists of a prior $\mu$ with two states in its support, $\Omega(\mu)=\left\{\omega_{1}, \omega_{2}\right\}$, each of which is equally likely. ${ }^{1}$ The support of the strategy comprises two posteriors, $\Gamma\left(Q_{\lambda^{*}}\right)=\left\{\gamma^{a}, \gamma^{b}\right\}$ :

$$
\gamma^{a}=\left(\begin{array}{c}
0.8 \\
0.2
\end{array}\right) \quad \gamma^{b}=\left(\begin{array}{c}
0.4 \\
0.6
\end{array}\right)
$$

and specifies $Q_{\lambda^{*}}\left(\gamma^{a}\right)=0.25$ and $Q_{\lambda^{*}}\left(\gamma^{b}\right)=0.75$. Actions $a$ and $b$ are chosen deterministically from $\gamma^{a}$ and $\gamma^{b}$ respectively, $q_{\lambda^{*}}\left(a \mid \gamma^{a}\right)=q_{\lambda^{*}}\left(b \mid \gamma^{b}\right)=1$.

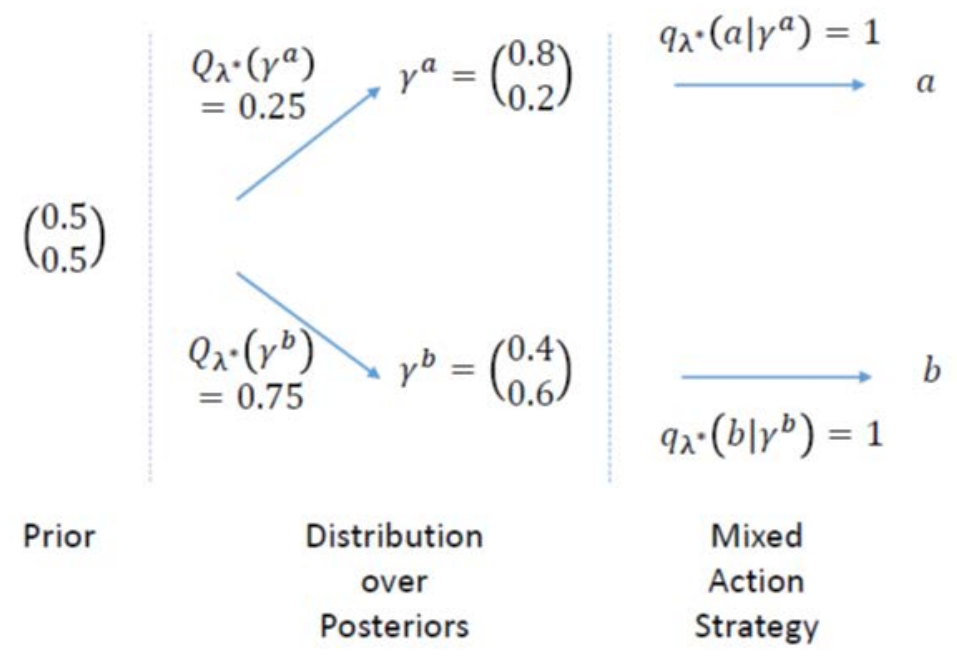

Figure 1: Strategy $\lambda^{*}$

\footnotetext{
${ }^{1}$ We use the notation

$$
\gamma=\left(\begin{array}{c}
\gamma\left(\omega_{1}\right) \\
\gamma\left(\omega_{2}\right)
\end{array}\right)
$$
}

to describe probability distributions. 


\section{$2.2 \quad$ Utility and Costs}

The goal of the DM is to maximize prize-based expected utility (EU) net of additively separable attention costs. Given $\lambda \in \Lambda$, prize based EU is computed in the standard manner,

$$
U(\lambda) \equiv \sum_{\gamma \in \Gamma\left(Q_{\lambda}\right)} \sum_{a \in A} Q_{\lambda}(\gamma) q_{\lambda}(a \mid \gamma) \bar{u}(\gamma, a)
$$

where $\bar{u}(\gamma, a)$ is expected utility conditional on the posterior $\gamma$

$$
\bar{u}(\gamma, a) \equiv \sum_{\omega \in \Omega(\mu)} \gamma(\omega) u(a, \omega)
$$

Attention costs for strategy $\lambda \in \Lambda(\mu)$ depend only on the distribution of posteriors $Q_{\lambda} \in \mathcal{Q}(\mu)$. We assume that inattention is always possible, and normalize its cost to zero. We allow for the possibility that some distributions of posteriors are infeasible by setting their costs to infinity. For example, there are interesting cases in which it is prohibitively costly to entirely rule out ex ante possible states, so that it is infeasible to choose posteriors on the boundary of $\Gamma(\mu)$.

Definition 4 We define $\mathcal{F}$ as the set of all priors and Bayes' consistent posterior distributions,

$$
\mathcal{F}=\{(\mu, Q) \mid \mu \in \Gamma, Q \in \mathcal{Q}(\mu)\} .
$$

We define $\mathcal{K}$ as the set of all attention cost functions $K: \mathcal{F} \rightarrow \overline{\mathbb{R}}$ such that $K\left(\mu, Q_{\lambda}\right)=0$ for $\lambda \in \Lambda^{I}(\mu)$.

\subsection{The Shannon Cost Function}

By far the best studied cost function that can be expressed directly in terms of priors and posteriors is the Shannon function, in which the costs are linear in the mutual information between prior and posteriors. It is standard that one can compute mutual information by comparing the Shannon entropy of the prior, $H(\mu)=-\sum_{\omega \in \Omega(\gamma)} \mu(\omega) \ln \mu(\omega)$, to the expected Shannon entropy of the posteriors. In translating this into an attention cost function, note that what is costly is increasing predictability, or reducing entropy. Given $(\mu, Q) \in \mathcal{F}$, the Shannon attention cost function $K_{\kappa}^{S}$ with multiplicative factor $\kappa>0$ is therefore specified as,

$$
K_{\kappa}^{S}(\mu, Q) \equiv \kappa\left[\sum_{\gamma \in \Gamma(Q)} Q(\gamma)[-H(\gamma)]-[-H(\mu)]\right]=\kappa\left[\sum_{\gamma \in \Gamma(Q)}-Q(\gamma) H(\gamma)+H(\mu)\right] .
$$

By way of illustration, consider attention strategy $\lambda^{*}$ from Figure 1 . Figure 2 records the probability of state $\omega_{1}$ on the horizontal axis. The Figure reflects the fact that Shannon entropy is strictly concave and symmetric around its maximized value at uniformity and that it is zero at the end-points of the interval ( $\operatorname{since} \lim _{x \downarrow 0} x \ln x=0$ ), at which it has unbounded derivative. Following (3), we shift up the negative of the entropy function, which is strictly convex, to zero at the prior of 0.5 . The cost of strategy $\lambda^{*}$ is then found as the height of the chord joining the points on the function corresponding to the two possible posterior likelihoods of $\omega_{1}(0.4$ and 0.8$)$ as it passes over the prior, as Figure 2 illustrates. 


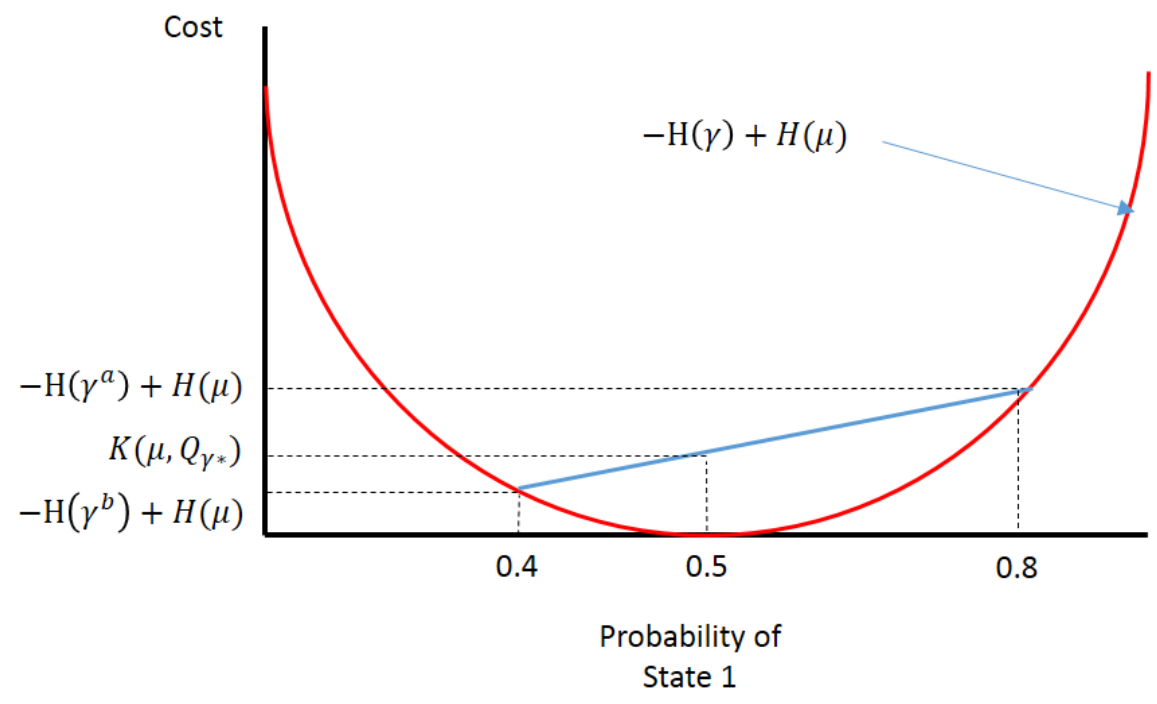

Figure 2: Cost of Strategy $\lambda^{*}$

Note that the Figure shows that all attentive strategies have strictly positive cost.

\subsection{PS Cost Functions}

The posterior-separable (PS) cost functions we study have the same form as (3), yet generalize the underlying measure of disorder, or "entropy", of the probability distribution over prior possible states of the world. The only properties that are retained relate to the strict convexity of this function and the specification of inattention as feasible and free.

Definition 5 An attention cost function is posterior-separable (PS), $K \in \mathcal{K}^{P S}$, if, given $\mu \in$ $\Gamma$, there exists a strictly convex function $T_{\mu}: \Gamma(\mu) \rightarrow \overline{\mathbb{R}}$, real-valued on $\tilde{\Gamma}(\mu)$, such that, given $Q \in \mathcal{Q}(\mu)$

$$
K(\mu, Q)=\sum_{\gamma \in \Gamma(Q)} Q(\gamma) T_{\mu}(\gamma)-T_{\mu}(\mu),
$$

and such that the optimal posterior set $\hat{\Gamma}(\mu \mid K)$,

$$
\hat{\Gamma}(\mu \mid K)=\left\{\gamma \in \Gamma \mid \exists(\mu, A) \in \mathcal{D} \text { and } \lambda \in \hat{\Lambda}(\mu, A \mid K) \text { with } \gamma \in \Gamma\left(Q_{\lambda}\right)\right\}
$$

is convex.

To clarify fine points in the definition, note that allowing $T_{\mu}$ to take infinite values for boundary posteriors both covers various interesting forms of entropy (see section 8.3) and simplifies our behavioral characterization. Strict convexity in this case means that, given distinct posteriors $\gamma_{1}, \gamma_{2}$ at which $T_{\mu}$ is real-valued (the set $\operatorname{dom} T_{\mu}$ in the notation of Rockafellar [1970] p. 23),

$$
T_{\mu}\left(\alpha \gamma_{1}+(1-\alpha) \gamma_{2}\right)<\alpha T_{\mu}\left(\gamma_{1}\right)+(1-\alpha) T_{\mu}\left(\gamma_{2}\right)
$$


all $\alpha \in(0,1)$ : hence dom $T_{\mu}$ itself is a convex set. Our insistence that $\hat{\Gamma}(\mu \mid K)$ is also convex avoids complications associated with possible non-existence of sub-differentials on the boundary. ${ }^{2}$

As noted in Section 9, functions of the PS form have featured in the literature on measures of the information content of experiments following Blackwell [1951]. A straight forward result with this functional form is that the strict convexity of $T_{\mu}$ ensures that the corresponding measure strictly respects the Blackwell partial ordering of information content (see Torgersen [1991]).

In addition to allowing for general convex cost functions, note that this definition allows costs to differ arbitrarily across priors, e.g. according to the cardinality of the state space. Subtraction of $T_{\mu}(\mu)$ is a normalization which ensures that inattentive strategies are free as per the general definition. Note that there are many different $T$ functions that give rise to precisely the same cost function. In particular, we show in the Appendix that $K$ is invariant to affine transforms of $T_{\mu}$ (Lemma 4.3).

\subsection{UPS Cost Functions}

While the PS case allows for arbitrary dependence of the cost function on the prior, the Shannon model does not exploit this freedom. Given distinct priors $\mu, \mu^{\prime} \in \Gamma$, the function $T_{\mu}(\gamma)$ and $T_{\mu^{\prime}}(\gamma)$ can be written in a manner that is independent of the prior. A fine point relates to the possibly infinite costs of ruling out ex ante possible states. Note that even with Shannon cost functions, the incremental cost of fully ruling out any prior possible state is unbounded at the margin. This means that there is not full independence between the prior and the cost of the corresponding posterior. However this dependence is limited. We can cover all such cases by insisting on a common $T$ function only for posteriors consistent with optimality.

Definition 6 A PS cost function $K \in \mathcal{K}^{P S}$ is uniformly posterior-separable (UPS), $K \in$ $\mathcal{K}^{U P S}$, if there exists a strictly convex function $T: \Gamma \rightarrow \mathbb{R}$ such that,

$$
K(\mu, Q)=\sum_{\gamma \in \Gamma(Q)} Q(\gamma) T(\gamma)-T(\mu)
$$

all $(\mu, Q) \in \mathcal{F}$ such that $Q \in \hat{\mathcal{Q}}(\mu \mid K) \equiv \mathcal{Q}(\mu) \cap \Delta(\hat{\Gamma}(\mu \mid K))$.

Examples of cost functions which fall into the UPS category are those based on alternative measures of entropy, such as that introduced by Tsallis [1988]. We discuss the relationship between Tsallis and Shannon costs in Section 8.3.

\section{$3 \quad$ PS Models, Optimal Strategies, and Lagrangians}

In this section we identify optimal strategies using Lagrangian methods. We develop the geometric intuition in the body of the text, with technical arguments in Appendix 1.

\footnotetext{
${ }^{2}$ In general, the set of posteriors at which sub-differentials exist (dom $\partial T_{\mu}$ in the notation of Rockafellar [1970], p. 227) need not be convex in particular contrived cases. Our results are most straight forward with $\hat{\Gamma}(\mu \mid K)$ convex, which holds for all standard forms of entropy. While both are convex, note that $\hat{\Gamma}(\mu \mid K)$ may be a strict subset of dom $T_{\mu}$. For example, the Shannon cost function is real-valued on the convex set $\Gamma(\mu)$, while $\hat{\Gamma}\left(\mu \mid K_{\kappa}^{S}\right)$ comprises only interior posteriors, $\hat{\Gamma}\left(\mu \mid K_{\kappa}^{S}\right)=\tilde{\Gamma}(\mu)$.
} 


\subsection{Optimal Strategies}

The value of strategy $\lambda \in \Lambda(\mu)$ is computed based on additive separability of prize utility and attention costs.

$$
V(\mu, \lambda \mid K) \equiv U(\lambda)-K\left(\mu, Q_{\lambda}\right) .
$$

The value function and corresponding optimal strategies are then defined as:

$$
\begin{aligned}
\hat{V}(\mu, A \mid K) & \equiv \sup _{\{\lambda \in \Lambda(\mu, A)\}} V(\mu, \lambda \mid K) \\
\hat{\Lambda}(\mu, A \mid K) & \equiv\{\lambda \in \Lambda(\mu, A) \mid V(\mu, \lambda \mid K)=\hat{V}(\mu, A \mid K)\} .
\end{aligned}
$$

\section{$3.2 \quad$ Net Utility}

There are Lagrangian methods of characterizing optimal strategies in the PS model. Yet the fact that costs can depend on the prior in the PS model gives rise to certain notational complexities. Hence for expository purposes, we focus on the UPS case, noting at the end that the approach generalizes to the PS case.

The key geometric observation is that the value of any given strategy, modulo the normalizing factor $T(\mu)$, can be decomposed into action specific net utilities, $N^{a}(\gamma)$,

$$
N^{a}(\gamma) \equiv \bar{u}(\gamma, a)-T(\gamma)
$$

To confirm, note that since $\sum_{a \in A} q_{\lambda}(a \mid \gamma)=1$ all $\gamma \in \Gamma\left(Q_{\lambda}\right)$,

$$
\begin{aligned}
V(\mu, \lambda \mid K)+T(\mu) & =\sum_{\gamma \in \Gamma\left(Q_{\lambda}\right)} \sum_{a \in A} Q_{\lambda}(\gamma) q_{\lambda}(a \mid \gamma) \bar{u}(\gamma, a)-\sum_{\gamma \in \Gamma\left(Q_{\lambda}\right)} Q_{\lambda}(\gamma) \sum_{a \in A} q_{\lambda}(a \mid \gamma) T(\gamma) \\
& =\sum_{\gamma \in \Gamma\left(Q_{\lambda}\right)} \sum_{a \in A} Q_{\lambda}(\gamma) q_{\lambda}(a \mid \gamma) N^{a}(\gamma) .
\end{aligned}
$$

Hence optimal strategies can be identified as those that maximize the weighted averages of net utilities.

The net utility approach has simple geometric content. In Figure 3 we illustrate action-specific net utilities in a simple two-state case with $\Omega(\mu)=\left\{\omega_{1}, \omega_{2}\right\}$ and $\mu\left(\omega_{1}\right)=0.5$. The probability of state 1 is on the horizontal axis. The red, dashed line graphs $T(\gamma)$ as a function of $\gamma\left(\omega_{1}\right)$. The green line represents the prize-based expected utility of an action $a$ in which we have assumed that: $u\left(a, \omega_{1}\right)=1$ and $u\left(a, \omega_{2}\right)=0$. To compute net utility we simply subtract the cost from the benefit (for clarity in the Figure we illustrate $N^{a}(\gamma)+T(\mu)$ which allows us to see the tangency of net costs with gross costs when $\gamma=\mu$ ). The result is the blue line in the Figure. Note that since net utility is the difference between a line and a strictly convex function, it is strictly concave. 


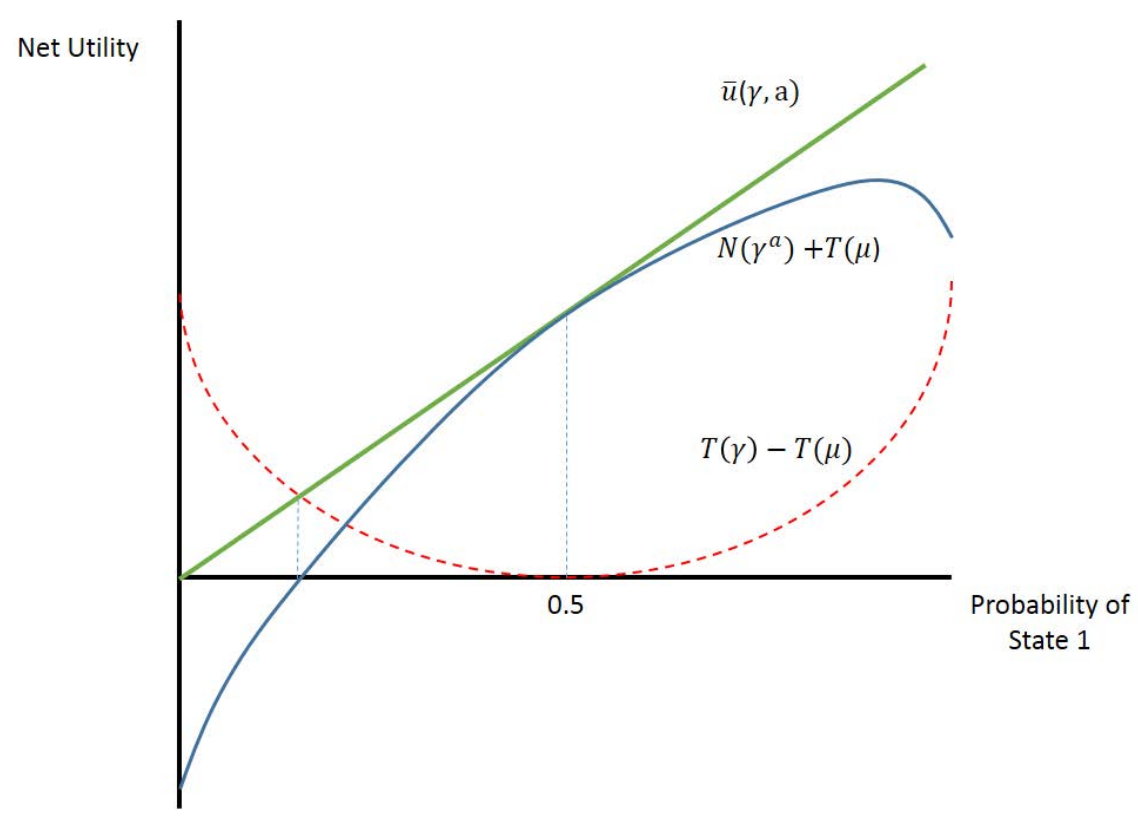

Figure 3: Net Utility of Action $a$.

Figure 4 illustrates net utilities for a decision problem $(\mu, A)$ with two equiprobable states and two actions, $A=\{a, b\}$. The second action is the mirror image of the first, with $u\left(b, \omega_{1}\right)=0$ and $u\left(b, \omega_{2}\right)=1$. We illustrate in the Figure computation of the net utility of strategy $\lambda^{*}$. Precisely as when computing the cost, the value is found by joining the points on the net utility function corresponding to possible posteriors with a chord, and finding the value of the chord as it passes over the prior. Thinking of all such chords identifies optimal strategies as defined by the posteriors that support the highest chord passing over the prior. In Figure 4 posteriors $\hat{\gamma}^{a}$ and $\hat{\gamma}^{b}$ have this property, and so form the support of an optimal strategy for this decision problem. Note that our example strategy $\lambda^{*}$ is non-optimal, since the corresponding chord passes strictly below the top 
chord.

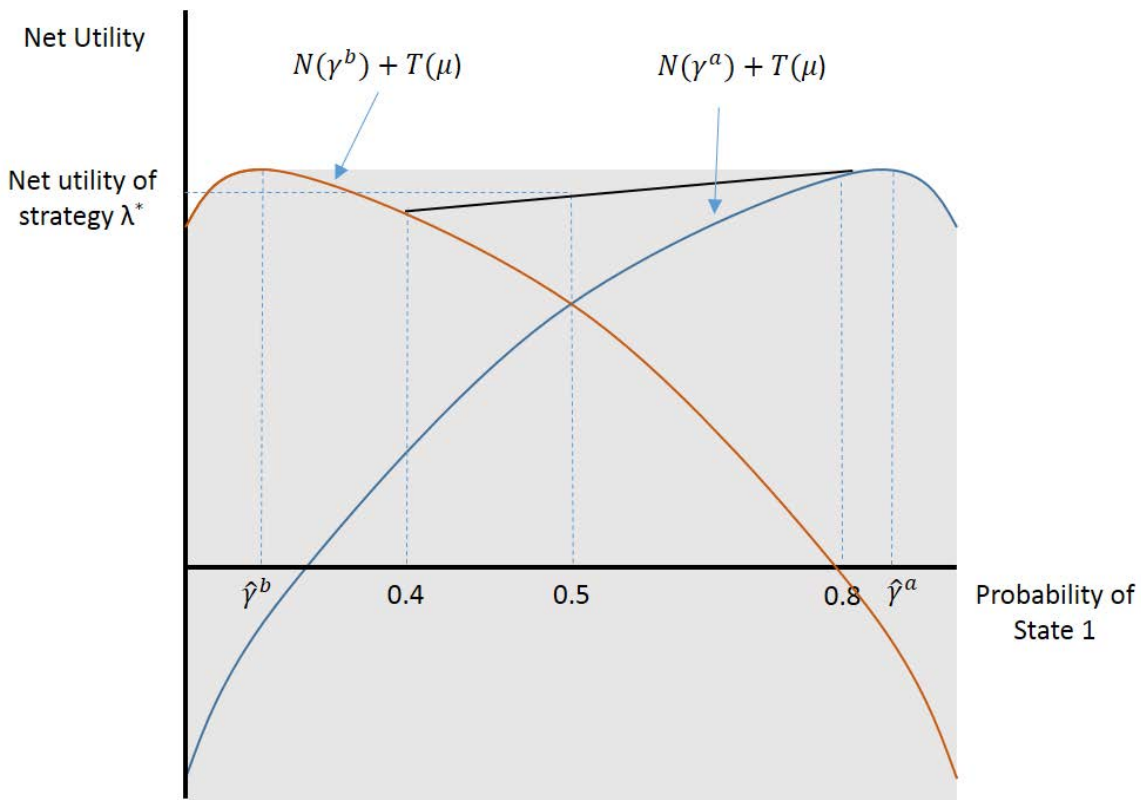

Figure 4: Net Utility of Strategy $\lambda^{*}$

\subsection{Lagrange Multipliers and the PS model}

The shaded area in Figure 4 is the lower epigraph of the concavified net utility function, defined as the minimal concave function that majorizes all net utilities (Rockafellar [1970]). The applicability of Lagrangian methods rests on the fact that the lower epigraph is always a convex set. This is geometrically clear in the simple case illustrated in Figure 4, and applies quite generally. Indeed, the same geometric approach works not only for UPS cost functions, but also for PS cost functions, in which net utilities are specific to the prior. For PS cost functions, we fix the prior $\mu$ and again define action-specific net utilities as $N_{\mu}^{a}(\gamma)$,

$$
N_{\mu}^{a}(\gamma) \equiv \bar{u}(\gamma, a)-T_{\mu}(\gamma)
$$

The key geometric observation is that one can still compute optimal strategies by appropriately averaging these action and prior specific net utilities. Hence identical convex analytic methods apply.

The geometric approach in Figure 4 is completely general. There is one important point to note in so generalizing, which derives from the adding up constraint on probabilities. Given this constraint, Figure 4 represents a two-dimensional state space in one dimension. This transformation is of great general value. Given $\mu \in \Gamma$ with $|\Omega(\mu)|=J$, we transform $\Omega(\mu)$ into the equivalent subspace of $\mathbb{R}^{J-1}$. To simplify, we give all states distinct integer labels $1 \leq j \leq J$, and let $\Gamma^{J-1}$ 
denote the corresponding space of probability distributions:

$$
\Gamma^{J-1}=\left\{\mu \in \mathbb{R}_{+}^{J-1} \mid \sum_{j=1}^{J-1} \mu(j) \leq 1\right\} ;
$$

with $\mu(J)=1-\sum_{j=1}^{J-1} \mu(j)$ left as implicit.

In Appendix 2 we establish a "Lagrangian" lemma that shows that there is always a supporting hyperplane to the lower epigraph of the convexified net utility function (Lemma 2.6). The analytic translation of this geometrically clear result is that optimal attention strategies are characterized by Lagrange multipliers $\theta(j)$ conveying the change in net utility as each posterior $\gamma(j)$ for $1 \leq j \leq J-1$ is raised at the expense of reducing $\gamma(J)$. The Lagrange multipliers define the slope of the supporting hyperplane at the optimum. All chosen actions have net utilities that lie on this hyperplane at the corresponding chosen posterior, while no net utility function breaches the hyperplane for any posterior.

Lagrangean Lemma: Given $K \in \mathcal{K}^{P S}$ and $(\bar{\mu}, A) \in \mathcal{D}, \lambda \in \hat{\Lambda}(\bar{\mu}, A \mid K)$ if and only if $\exists \theta \in \mathbb{R}^{J-1}$ s.t.,

$$
N_{\bar{\mu}}^{a}(\gamma)-\sum_{j=1}^{J-1} \theta(j) \gamma(j) \leq \sup _{a^{\prime} \in A, \gamma^{\prime} \in \Gamma(\bar{\mu})} N_{\bar{\mu}}^{a^{\prime}}\left(\gamma^{\prime}\right)-\sum_{j=1}^{J-1} \theta(j) \gamma^{\prime}(j),
$$

all $\gamma \in \Gamma(\bar{\mu})$ and $a \in A$, with equality if $\gamma \in \Gamma\left(Q_{\lambda}\right)$ and $q_{\lambda}(a \mid \gamma)>0$.

Note that this lemma characterizes optimal strategies, and opens up standard methods of model solution. In addition, it conveys important qualitative features of the behavior implied by PS and UPS models. We return to this in later sections.

\section{SDSC and Representations}

In this section we introduce the data set and the sought after representations.

\subsection{State Dependent Stochastic Choice Data}

The key question in applied work on attention is the extent to which DMs internalize the actual decision making environment in which they find themselves. Do they notice whether or not a sales tax is included in the price paid at the register (Chetty et al. [2009])? Do they notice fluctuating prices of the same good in a supermarket (Matějka [2015])? Essentially all such situations can be captured using the general model above, by appropriately specifying available actions, the various factors (states of the world) that determine their payoffs, and prior beliefs about how likely is each such state.

Our goal is to specify observable patterns in choice data that narrow down the theories of inattentive choice. Before we begin, however, we must first specify exactly what sort of data is sufficient for this task. An important first point is that standard stochastic choice data, in which one only observes the unconditional likelihood of each choice, is fundamentally inadequate for capturing 
attentional constraints. To see this, consider the two action decision problem illustrated in Figure 4. Note that the symmetry of the decision problem implies that the optimal strategy results in each action being chosen equally often. In the particular strategy chosen, this reflects partial information. Yet the same unconditional probabilities are also consistent with perfect information, with each action chosen precisely when it is optimal. These probabilities are also consistent with completely inattentive choice, with a fair coin flipped to decide which action is taken. Unconditional choice probabilities in no way reflect the extent to which behavioral patterns are impacted by reality. One must also know how well the action suited reality.

As first noted by Block and Marschak [1960] (p. 98-99), the way forward lies in realistically enriching the ideal behavioral data available to an ideal observer (IO), such as an econometrician or economic theorist, in which of costs of attention are to be identified. The key to our data enrichment is the observation that the information constraints that impact the DM do not apply to the IO. For example, while the DM may have difficulty assessing whether or not a sales tax is included in the purchase price or what the actual price of each good is in a supermarket, the IO with access to the underlying reality does not. In defining our data, we therefore specify that the IO observes both the state of the world as well as the action.

In formal terms, our behavioral data set is state dependent stochastic choice (SDSC) data, as in Caplin and Martin [2015] and Caplin and Dean [2015]. We specify both states and actions as being fully observed by the IO. We further specify our IO as able to watch this DM facing this same decision infinitely often, with precisely this strategy used each time. ${ }^{3}$ For the IO to treat repeated observations of the DM as deriving from the same decision problem implies that the set of available actions, $A$, is the same. It requires also that the DM is seen as having the same prior $\mu$ over possible states of the world. We assume that the IO then observes the full distribution of actual state realizations and action choices. In terms of interpreting the data as revealing of patterns of attentional choice, we make the simplifying assumption that there are common probability assessments between IO and DM. We call this "rational expectations" with which it has spiritual commonalities.

A key observation is that rationality of expectations enables the IO to infer the DM's presumed prior as the actual proportion of times each state is realized. We therefore treat the prior itself as observable in specifying our behavioral data set in its most general form.

Definition 7 Given $(\mu, A) \in \mathcal{D}$, we define state dependent stochastic choice (SDSC) data as mapping from possible states to action probabilities,

$$
\mathcal{P}(\mu, A) \equiv\{P: \Omega(\mu) \rightarrow \Delta(A)\}
$$

with $P(a \mid \omega)$ the probability of action a in state $\omega$. We define $\mathcal{P}(\mu)$ as the union over all corresponding decision problems and $\mathcal{P} \equiv \cup_{\mu \in \Gamma} \mathcal{P}(\mu)$.

Implicit in this definition is the assumption that the expected utility function of the DM is part of the data. One could readily replace this assumption with an enrichment of the data set that allowed for utilities to be recovered from behavior, as discussed in Caplin and Dean [2015].

\footnotetext{
${ }^{3}$ In practice one might apply a model of this form to a population rather than an individual, as in the literature on discrete choice following McFadden [2005].

${ }^{4}$ One could replace the "Savage style" actions we use in this paper with "Anscombe-Aumann" acts that map states
} 
While only recently introduced in economics, SDSC data has a long and storied history in psychometrics. The Weber-Fechner laws, which are based on corresponding data, identify regularities in how well humans perceive objective differences in the strength of various external stimuli.

There are two key differences between our approach and the standard psychometric approach. First, we follow classical economic logic, so that the stimuli are levels of utility, or reward. Second, we model perceptual effort as chosen in light of potential rewards. Given this, we will show that rich behavioral data has patterns in it that fully reveal costs of accurately recognizing external reward stimuli.

\subsection{From Strategy to Data}

We illustrate in Figure 5 how seeing data on states and actions captures the behavioral imprint of our running example, strategy $\lambda^{*}$. Given the assumed rationality of expectations, the subjective probabilities of the DM agree with the data frequencies as seen by the IO. What the IO will then see is a joint distribution of states and actions with precise probabilities determined by the prior, the posteriors, and the mixed action strategy.

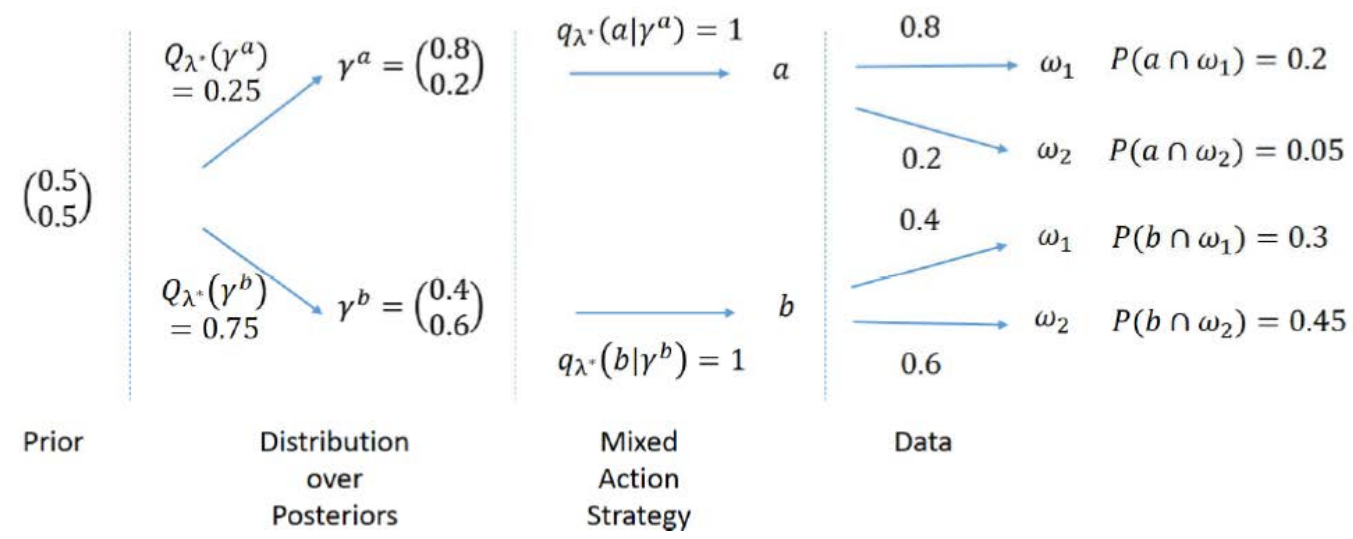

Figure 5: Data Generated by Strategy $\lambda^{*}$

The fact that action $a$ is chosen if and only if the DM receives $\gamma^{a}$, and that $Q_{\lambda^{*}}\left(\gamma^{a}\right)=0.25$ means that action $a$ will be chosen $25 \%$ of the time (and $b$ the remaining $75 \%$ of the time). Because $\gamma^{a}$ is associated with an $80 \%$ probability of $\omega_{1}$ (and a $20 \%$ probability of $\omega_{2}$ ), the resulting joint probability of $a$ and $\omega_{1}$ is $20 \%$. All other joint probabilities can be calculated in a similar way, as shown in Figure 5. These joint probabilities can be converted into conditional probabilities using Bayes' rule, giving the SDSC $P^{*}$ associated with $\lambda^{*}$ :

$$
\begin{aligned}
& P^{*}\left(a \mid \omega_{1}\right)=0.4 ; \quad P^{*}\left(b \mid \omega_{1}\right)=0.6 \\
& P^{*}\left(a \mid \omega_{2}\right)=0.1 ; \quad P^{*}\left(b \mid \omega_{2}\right)=0.9 .
\end{aligned}
$$

of the world to probability distributions over the prize space. Assuming the DM does maximize expected utility, $u$ could then be recovered by observing choices over degenerate acts (i.e. acts whose payoffs are state independent). 
This method for generating data from strategies is more general. Following the logic of Figure 5, we translate each strategy $\lambda \in \Lambda(\mu, A)$ into its observable counterpart in SDSC data, $\mathbf{P}_{\lambda}$, assuming rational expectations. With this notation, note that $P^{*}=\mathbf{P}_{\lambda^{*}}$.

Definition 8 Given $\lambda \in \Lambda(\mu, A)$ we define the generated $S D S C$ data $\mathbf{P}_{\lambda}: \Omega(\mu) \rightarrow \Delta(A)$ and the corresponding action choice probabilities $\mathbf{P}_{\lambda}(a)$ on $a \in \mathcal{A}(\lambda)$ by:

$$
\begin{aligned}
\mathbf{P}_{\lambda}(a \mid \omega) & =\frac{\sum_{\gamma \in \Gamma\left(Q_{\lambda}\right)} Q_{\lambda}(\gamma) q_{\lambda}(a \mid \gamma) \gamma(\omega)}{\mu(\omega)} \\
\mathbf{P}_{\lambda}(a) & =\sum_{\gamma \in \Gamma\left(Q_{\lambda}\right)} Q_{\lambda}(\gamma) q_{\lambda}(a \mid \gamma) .
\end{aligned}
$$

\subsection{Choice Correspondence and Representations}

In the idealized data set that we consider, SDSC data is available for all decision problems. As indicated, and as in Caplin and Dean [2015], we assume that the IO knows all details of the decision problem faced by the DM, which includes the prior and the payoffs to all available actions. For technical reasons, it simplifies the statement of our representation theorems to imagine that the IO sees a data set that is deep as well as broad. It specifies for each decision problem a corresponding set of qualifying SDSC functions - i.e. all such functions used by the DM in that decision problem. Following Richter [1966], this is in the spirit of standard choice analysis based on a correspondence mapping a choice set to a subset of suitable alternatives. $\mathcal{C}$ is the set of such data sets:

$$
\mathcal{C} \equiv\left\{C: \mathcal{D} \rightarrow 2^{\mathcal{P}} / \emptyset \mid C(\mu, A) \subset \mathcal{P}(\mu, A)\right\}
$$

This level of artificiality turns out to be substantively irrelevant. We discuss in Section 8 how our results extend to cases in which one sees only a selection from this data correspondence.

We say that a data set $C$ has a costly information representation based on a cost function $K$ if the observed SDSC data $C(\mu, A)$ corresponding to each decision problem $(\mu, A)$ coincides with the SDSC data $\mathbf{P}_{\lambda}$ generated by optimal strategies $\lambda \in \hat{\Lambda}(\mu, A \mid K)$.

Definition 9 Data set $C \in \mathcal{C}$ has a costly information representation $(C I R)$ based on $K \in \mathcal{K}$ if, for all $(\mu, A) \in \mathcal{D}$,

$$
C(\mu, A)=\left\{\mathbf{P}_{\lambda} \in \mathcal{P} \mid \lambda \in \hat{\Lambda}(\mu, A \mid K)\right\} \equiv \hat{P}(\mu, A \mid K)
$$

1. It has a posterior-separable (PS) representation it is has a $C I R K \in \mathcal{K}^{P S}$.

2. It has a uniformly posterior-separable (PS) representation it is has a $C I R K \in \mathcal{K}^{U P S}$.

3. It has a Shannon representation if it has a CIR $K=K_{\kappa}^{S}$ for $\kappa>0$.

\subsection{The Revealed Strategy}

Caplin and Dean [2015] show that, while there is a multiplicity of strategies that could have generated any SDSC data, there is always a unique least Blackwell informative strategy consistent 
with the data. The first step in constructing this strategy is to identify with each chosen action $a$ the corresponding "revealed posterior" $\bar{\gamma}_{P}^{a}$. This treats the action as chosen at one and only one posterior which can be inferred from our behavioral data using Bayes' rule. Building on this, the "revealed posterior-based strategy" is the least Blackwell informative strategy consistent with the data. As such, it is the least costly for all our PS cost functions. It follows that for the class of models we consider in this paper, optimality implies that the revealed attention strategy is used by the DM in each decision problem.

Definition 10 Given $(\mu, A) \in \mathcal{D}, P \in \mathcal{P}(\mu, A)$, and $a \in A$, we define revealed action probability $P(a)=\sum_{\omega \in \Omega(\mu)} \mu(\omega) P(a \mid \omega)$. We define $\mathcal{A}(P)$ as the actions chosen with positive probability. If $a \in \mathcal{A}(P) \subset A$, we define also revealed posterior $\bar{\gamma}_{P}^{a} \in \Gamma(\mu)$

$$
\bar{\gamma}_{P}^{a}(\omega)=\frac{\mu(\omega) P(a \mid \omega)}{P(a)}
$$

with $\Gamma(P)$ the union across $a \in \mathcal{A}(P)$. The revealed posterior-based attention strategy $\boldsymbol{\lambda}(P)=$ $\left(\mathbf{Q}_{P}, \mathbf{q}_{P}\right) \in \Lambda(\mu, A)^{5}$ is defined by $\Gamma\left(\mathbf{Q}_{P}\right)=\cup_{a \in \mathcal{A}(P)} \bar{\gamma}_{P}^{a}$ and:

$$
\begin{aligned}
\mathbf{Q}_{P}(\gamma) & =\sum_{\left\{a \in \mathcal{A}(P) \mid \bar{\gamma}_{P}^{a}=\gamma\right\}} P(a) ; \\
\mathbf{q}_{P}(a \mid \gamma) & =\left\{\begin{array}{cc}
\frac{P(a)}{\mathbf{Q}_{P}(\gamma)} & \text { if } \bar{\gamma}_{P}^{a}=\gamma ; \\
0 & \text { if } \bar{\gamma}_{P}^{a} \neq \gamma
\end{array}\right.
\end{aligned}
$$

To illustrate construction of the revealed attention strategy, consider the data set $P^{*}=\mathbf{P}_{\lambda^{*}}$. The revealed posterior associated with the choice of action $a$ is,

$$
\bar{\gamma}_{P^{*}}^{a}\left(\omega_{1}\right)=\frac{\mu\left(\omega_{1}\right) P^{*}\left(a \mid \omega_{1}\right)}{P^{*}(a)}=\frac{0.5 \times 0.4}{0.25}=0.8 .
$$

Similarly

$$
\bar{\gamma}_{P^{*}}^{a}\left(\omega_{2}\right)=0.2 ; \bar{\gamma}_{P^{*}}^{b}\left(\omega_{1}\right)=0.4 ; \text { and } \bar{\gamma}_{P^{*}}^{b}\left(\omega_{2}\right)=0.6
$$

We can then calculate the revealed strategy as involving

$$
\begin{aligned}
Q_{P^{*}}\left(\bar{\gamma}_{P^{*}}^{a}\right) & =P^{*}(a)=\mu\left(\omega_{1}\right) P^{*}\left(a \mid \omega_{1}\right)+\mu\left(\omega_{2}\right) P^{*}\left(a \mid \omega_{2}\right) \\
& =0.5 *(0.4+0.1)=0.25
\end{aligned}
$$

Hence,

$$
Q_{P^{*}}\left(\bar{\gamma}_{P^{*}}^{b}\right)=P^{*}(b)=0.75
$$

Furthermore,

$$
q_{P^{*}}\left(a \mid \bar{\gamma}_{P^{*}}^{a}\right)=1=q_{P^{*}}\left(b \mid \bar{\gamma}_{P^{*}}^{b}\right) .
$$

Note in this case that $\lambda^{*}$ is in fact the revealed strategy associated with data set $\mathbf{P}_{\lambda^{*}}=P^{*}$,

$$
\lambda^{*}=\boldsymbol{\lambda}\left(P^{*}\right)=\boldsymbol{\lambda}\left(\mathbf{P}_{\lambda^{*}}\right) .
$$

While this does not hold for arbitrary strategies, it is general for data observed in our representa-

\footnotetext{
${ }^{5}$ See Appendix 1 for direct confirmation that $\boldsymbol{\lambda}(P) \in \Lambda(\mu, A)$.
} 
tions, as discussed in Caplin and Dean [2015]. Appendix 2 contains this result together with other general results that link strategies revealed by data in costly information representations with the SDSC data generated by optimal strategies.

\section{Compression and the Shannon Model}

Having introduced the key model elements and data-related definitions, we turn now to the results themselves. In this section we start with a data set having a UPS representation and identify additional behavioral restrictions that make this a Shannon representation. As the definitions show, the UPS form allows for a general convex function $T(\gamma)$, while Shannon restricts $T(\gamma)$ to a particular one parameter family, $T(\gamma)=\kappa \ln (\gamma)$. This restriction on $T$ implies many qualitative restrictions on behavior. For example, there are strong symmetry properties, so that behavior must indicate that all states individually are equally easy or difficult to perceive. There are also no complementarities, so that learning about one state makes it no easier (or more difficult) to learn about any separate state. There are also very strong smoothness properties and profound quantitative restrictions e.g. in terms of the response to payoff changes.

Our first theorem establishes that a single behavioral invariance axiom is enough to move us from a UPS representation to a Shannon representation, hence conveying all of these particular properties noted above and all others besides. This axiom insists that choices not change when payoff equivalent states are "compressed" into a single state. In the remainder of the section we first introduce this behavioral axiom intuitively. We then formalize it and state the main theorem. Finally, we sketch the proof. The proof itself, which is involved, is in Appendix 5.

\subsection{Basic Decision Problems and Basic Forms}

What precisely does it mean to say that payoffs alone matter? To specify, consider first decision problems in which all states are distinct in terms of payoffs, so that no two possible states have identical payoffs for all available actions. We call these "basic" decision problems.

Definition 11 Given $(\mu, A) \in \mathcal{D}$, a decision problem is basic, $(\mu, A) \in \mathcal{B} \subset \mathcal{D}$ if, given $\omega \neq \omega^{\prime} \in$ $\Omega(\mu)$, there exists $a \in A$ such that $u(a, \omega) \neq u\left(a, \omega^{\prime}\right)$.

Consider now a non-basic decision problem with three possible states: $\Omega(\mu)=\left\{\omega_{1}, \omega_{2}, \omega_{3}\right\}$ and two actions $A=\{a, b\}$. In this problem, states $\omega_{1}$ and $\omega_{2}$ are equivalent:

$$
\begin{aligned}
& u\left(a, \omega_{1}\right)=1, u\left(b, \omega_{1}\right)=0 ; \\
& u\left(a, \omega_{2}\right)=1, u\left(b, \omega_{2}\right)=0 ; \\
& u\left(a, \omega_{3}\right)=0, u\left(b, \omega_{3}\right)=1 .
\end{aligned}
$$

There are two obvious ways to shift all probability from the two equivalent states to one or the other of them. One way is to set $\bar{\mu}\left(\omega_{1}\right)=\mu\left(\omega_{1}\right)+\mu\left(\omega_{2}\right)$ and $\bar{\mu}\left(\omega_{2}\right)=0$, with $\bar{\mu}\left(\omega_{3}\right)=\mu\left(\omega_{3}\right)$. The alternative is to set $\hat{\mu}\left(\omega_{2}\right)=\mu\left(\omega_{1}\right)+\mu\left(\omega_{2}\right)$ and rule out state $\omega_{1}$. These priors associated with these two "basic forms" of $(\mu, A)$ are illustrated in Figure 6. 


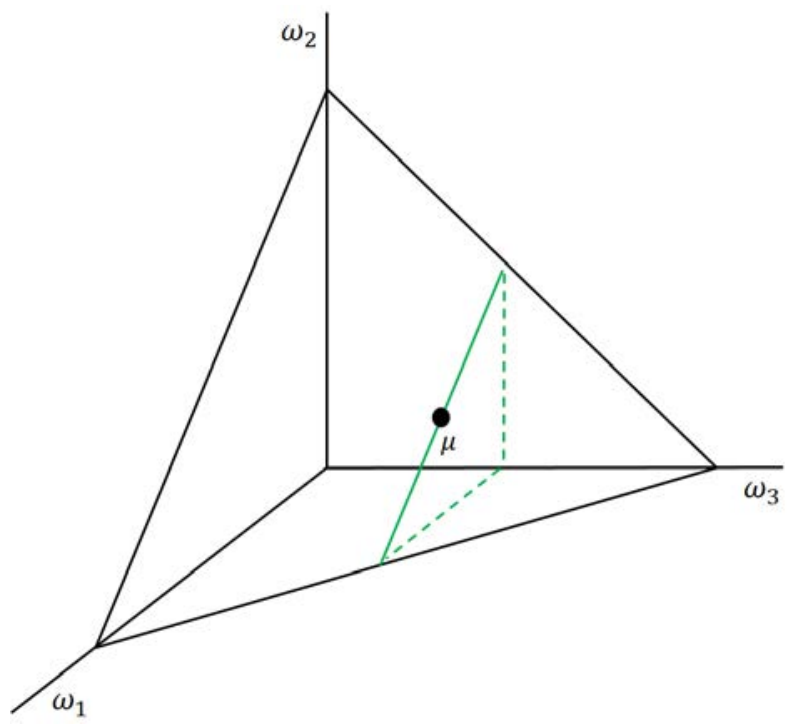

Figure 6: Basic Forms of Decision Problem

$(\mu, A)$

We now provide the general technical definitions.

Definition 12 We associate $(\mu, A) \in \mathcal{D}$ with a set of basic forms $(\bar{\mu}, A) \in \mathcal{B}(\mu, A) \subset \mathcal{B}$ by:

1. Partitioning $\Omega(\mu)$ into $L$ basic sets $\left\{\Omega^{l}(\mu)\right\}_{1 \leq l \leq L}$ comprising payoff equivalent states, so that, given $\omega \in \Omega^{l}(\mu)$ and $\omega^{\prime} \in \Omega^{m}(\mu)$,

$$
l=m \text { iff } u(a, \omega)=u\left(a, \omega^{\prime}\right) \text { all } a \in A \text {. }
$$

2. Labeling all possible states both by equivalence class and in order within each equivalence class:

$$
\Omega(\mu)=\left\{\omega_{i}^{l}|| 1 \leq i \leq I(l)=\left|\Omega^{l}(\mu)\right| \text { and } 1 \leq l \leq L\right\} .
$$

3. Selecting $\bar{\imath}(l) \in\{1, . ., I(l)\}$ all $l$ and defining $\bar{\Omega}(\mu)=\cup_{l=1}^{L} \omega_{\bar{\imath}(l)}^{l}$.

4. Defining $\bar{\mu} \in \Gamma(\mu)$ by setting

$$
\bar{\mu}\left(\omega_{i}^{l}\right)=\left\{\begin{array}{c}
\sum_{j=1}^{I(l)} \mu\left(\omega_{j}^{l}\right) \text { if } i=\bar{\imath}(l) ; . \\
0 \text { if } i \neq \bar{\imath}(l) .
\end{array}\right.
$$

Given $\bar{\imath}(l) \in\{1, . ., I(l)\}$ on $1 \leq l \leq L$, we say $(\bar{\mu}, A) \in \mathcal{B}(\mu, A)$ for $\bar{\imath}$. 


\subsection{Invariance Under Compression}

Note that there is no functional value for the DM in distinguishing between states that assign the same payoff to all actions. Hence an ideally designed machine for encoding states would not waste any of its scarce resources on this task. The stochastic structure of choice would not change if distinct yet payoff equivalent states were "compressed" into a single state.

Our Invariance under Compression axiom insists that patterns of choice are equivalent in all decision problems with a common basic form.

Axiom A1 Invariance under Compression (IUC): Given $(\bar{\mu}, A) \in B(\mu, A)$ for $\bar{\imath}$,

$$
P \in C(\mu, A) \Longleftrightarrow \exists \bar{P} \in C(\bar{\mu}, A) \text { s.t. } P\left(a \mid \omega_{i}^{l}\right)=\bar{P}\left(a \mid \omega_{\bar{\imath}(l)}^{l}\right),
$$

all $1 \leq i \leq I(l), 1 \leq l \leq L$ and $a \in A$.

We can illustrate the meaning of IUC using the example discussed above, in which the decision problem $(\mu, A)$ is such that $\Omega(\mu)$ has two basic sets: $\left\{\omega_{1}, \omega_{2}\right\}$ and $\left\{\omega_{3}\right\}$. Note first that IUC implies that, for any observed $P \in C(\mu, A)$ it must be the case that $P\left(a \mid \omega_{1}\right)=P\left(a \mid \omega_{2}\right)$ for all $a \in A$ : the DM must behave identically in any states that belong to the same basic set. Moreover, behavior in $(\mu, A)$ must be similar to behavior in the basic version of the problem. For example, given $\bar{\mu}\left(\omega_{1}\right)=\mu\left(\omega_{1}\right)+\mu\left(\omega_{2}\right), \bar{P} \in C(\bar{\mu}, A)$ if and only if $\bar{P}\left(a \mid \omega_{1}\right)=P\left(a \mid \omega_{1}\right)=P\left(a \mid \omega_{2}\right)$ for some $P \in C(\mu, A)$ and all $a \in A$. The fact that this also holds for the basic version of the problem in which $\hat{\mu}\left(\omega_{1}\right)=\mu\left(\omega_{1}\right)+\mu\left(\omega_{2}\right)$ means furthermore that behavior in the two basic versions of the problem must be the same: $\bar{P} \in C(\bar{\mu}, A)$ if and only if $\bar{P}\left(a \mid \omega_{1}\right)=\hat{P}\left(a \mid \omega_{2}\right)$ for some $\hat{P} \in C(\hat{\mu}, A)$. An immediate corollary is that, for any prior $\mu^{*}$ such that $\mu^{*}\left(\omega_{3}\right)=\mu\left(\omega_{3}\right)$ and $\Omega\left(\mu^{*}\right) \subset \Omega(\mu)$ it must be the case that $C(\mu, A)=C\left(\mu^{*}, A\right)$.

The key result is that the Shannon cost function alone among UPS cost functions satisfies this invariance axiom.

Theorem 1: Data set $C \in \mathcal{C}$ with a UPS representation has a Shannon representation if and only if it satisfies IUC.

\section{$5.3 \quad$ Necessity}

That IUC is necessary for a Shannon representation follows directly from the posterior-based characterization of the solution to the Shannon model. Caplin and Dean [2013] provide an "invariant likelihood ratio" condition for optimality. This states that $P \in C(\mu, A)$ is consistent with optimality for a cost function $K_{\kappa}^{S}$ if and only if:

1. Given $a, b \in \mathcal{A}(P)$,

$$
\frac{\bar{\gamma}_{P}^{a}(\omega)}{\exp (u(a, \omega) / \kappa)}=\frac{\bar{\gamma}_{P}^{b}(\omega)}{\exp (u(b, \omega) / \kappa)} \text { all } \omega \in \Omega(\mu)
$$


2. Given $a \in \mathcal{A}(P)$ and $c \in A \backslash \mathcal{A}(P)$,

$$
\sum_{\omega \in \Omega(\mu)}\left[\frac{\bar{\gamma}_{P}^{a}(\omega)}{\exp (u(a, \omega) / \kappa)}\right] \exp (u(c, \omega) / \kappa) \leq 1 .
$$

It is the fact that these conditions are invariant under the compression operation that establishes IUC as necessary for a Shannon representation, as formalized in Appendix 5.

\subsection{Guide to the Sufficiency Proof}

While the necessity proof is straight forward, the sufficiency proof is not. Theorem 1 establishes that IUC is profoundly powerful. It implies that, starting with behavior generated by a general strictly convex function, IUC plus one attentive choice pins down behavior in all decision problems. This follows since the attentive choice pins down the single parameter $\kappa>0$ in the Shannon function, leaving no more degrees of freedom.

Given the vast distance that the proof must travel to rule out all other forms of the cost function, it involves several stages that we elaborate on briefly here. The proof itself involves many corresponding lemmas that provide details.

One line of argument uses IUC to establish strong symmetry properties of the cost function: here the argument is direct. Two other key aspects of the proof take up issues of smoothness and functional form. In particular, there are strong differentiability and additive separability arguments. With these established, we identify a second order PDE that must be satisfied and that implies the Shannon form. The smoothness and separability arguments work in a fixed state space of cardinality 4 or higher. The final step in the proof involves using IUC to link cost functions across dimensions and to iterate down to dimensions below four. We briefly outline what is accomplished in each stage, leaving the full treatment to the Appendix.

\subsubsection{Symmetry}

The first step in the proof is to introduce and demonstrate the powerful symmetry implications of IUC. The definition of symmetry in beliefs is direct: $\gamma_{1}, \gamma_{2} \in \Gamma$ are symmetric, $\gamma_{1} \sim_{\Gamma} \gamma_{2}$, if there exists a bijection $\sigma: \Omega\left(\gamma_{1}\right) \rightarrow \Omega\left(\gamma_{2}\right)$ such that, for all $\omega \in \Omega\left(\gamma_{1}\right)$,

$$
\gamma_{1}(\omega)=\gamma_{2}(\sigma(\omega))
$$

Correspondingly, the strictly convex function $T: \Gamma \longrightarrow \mathbb{R}$ is symmetric if,

$$
\gamma_{1} \sim_{\Gamma} \gamma_{2} \Longrightarrow T\left(\gamma_{1}\right)=T\left(\gamma_{2}\right)
$$

A sequence of results establishes that IUC implies symmetry of the $T$ function in a UPS representation (Lemma 5.7).

Symmetric Cost Lemma: Given $C \in \mathcal{C}$ satisfying Axiom A1, any function $T: \Gamma \longrightarrow \mathbb{R}$ in a UPS representation $K(Q)=\sum_{\Gamma(Q)} Q(\gamma) T(\gamma)$ must be symmetric. 
Intuitively, Axiom A1 implies that relabeling the states cannot affect choice. To see this consider two basic decision problems $\left(\mu_{1}, A_{1}\right)$ and $\left(\mu_{2}, A_{2}\right)$ that are symmetric in the sense that $\mu_{1}(\omega)=$ $\mu_{2}(\sigma(\omega))$ for each $\omega \in \Omega\left(\gamma_{1}\right)$ and such that, for each $a \in A_{1}$, there exists $b \in A_{2}$ such that $u(a, \omega)=u(b, \sigma(\omega))$ where the implied mapping between $A_{1}$ and $A_{2}$ is bijective. Now consider a third problem $\left(\mu_{3}, A_{3}\right)$ which involves replicating $\left(\mu_{1}, A_{1}\right)$ and $\left(\mu_{2}, A_{2}\right)$ on a set of states $\Omega\left(\mu_{3}\right)$ disjoint from $\Omega\left(\mu_{1}\right) \cup \Omega\left(\mu_{2}\right)$, and then consider the problem $\left(\frac{\mu_{1}}{3}+\frac{\mu_{2}}{3}+\frac{\mu_{3}}{2}, A_{1} \cup A_{2} \cup A_{3}\right)$. ( $\left.\mu_{1}, A_{1}\right)$, $\left(\mu_{2}, A_{2}\right)$, and $\left(\mu_{3}, A_{3}\right)$ are all basic versions of this last problem and therefore the SDSC data generated by $\left(\mu_{1}, A_{1}\right)$ and $\left(\mu_{2}, A_{2}\right)$ is similar to the SDSC data generated by $\left(\mu_{3}, A_{3}\right)$ and hence each is similar to the other. It is a small step from this observation to the Symmetric Cost Lemma.

\subsubsection{Differentiability}

As noted above, much of the proof involves working within a fixed state space $\tilde{\Omega} \subset \Omega$ of cardinality $J \geq 4$, with the states indexed by $j$. Recall that $\tilde{\Gamma}$ comprise the interior posteriors with $\Omega(\gamma)=\tilde{\Omega}$ and we correspondingly let $\tilde{T}$ be the restriction of $T$ to $\tilde{\Gamma}$. By symmetry, the form of this function depends only on the cardinality $J$.

Given $\gamma \in \tilde{\Gamma}$ and any pair of states $i \neq j$ we define the one-sided derivative in direction $j i, \tilde{T}_{\overrightarrow{j i}}(\gamma)$, as the directional derivative associated with increasing the $i$ th coordinate and equally reducing the $j$ th:

$$
\tilde{T}_{\overrightarrow{j i}}(\gamma)=\lim _{\epsilon \downarrow 0} \frac{\tilde{T}\left(\gamma+\epsilon\left(e_{i}-e_{j}\right)\right)-\tilde{T}(\gamma)}{\epsilon} ;
$$

where $e_{k} \in \mathbb{R}^{J}$ is the corresponding unit vector. ${ }^{6}$

Since $\tilde{T}$ is convex, we know that $\tilde{T}_{\overrightarrow{j i}}(\gamma)$ exists. We define also the two-sided derivative in direction $j i, \tilde{T}_{(j i)}$, by:

$$
\tilde{T}_{(j i)}(\gamma)=\lim _{\epsilon \rightarrow 0} \frac{\tilde{T}\left(\gamma+\epsilon\left(e_{i}-e_{j}\right)\right)-\tilde{T}(\gamma)}{\epsilon} .
$$

While in principle the two-sided derivative need not exist, we show that it always does (Lemma 5.32). The proof makes heavy use of results in Rockafellar [1970] and the profound structure that IUC conveys. The proof is carried out in stages, interactively with the proof of full additive separability, discussed further below.

With $\tilde{T}_{(j i)}(\gamma)$ existing always, we can define cross directional derivatives of $\tilde{T}$. Given $\gamma \in \tilde{\Gamma}$ and any two pairs of states $i \neq j$ and $k \neq l$, we define the corresponding cross derivative of $\tilde{T}_{(j i)}$ in direction $l k$ as the corresponding (two-sided) directional derivative,

$$
\tilde{T}_{(j i)(l k)}(\gamma)=\lim _{\epsilon \rightarrow 0} \frac{\tilde{T}_{(j i)}\left(\eta+\epsilon\left(e_{k}-e_{l}\right)\right)-\tilde{T}_{(j i)}(\eta)}{\epsilon}
$$

Again, we show that these cross-derivatives exist everywhere in $\tilde{\Gamma}$ (Lemma 5.36).

\footnotetext{
${ }^{6}$ This is defined in Rockafellar [1970] as the directional derivative of $\tilde{T}$ at $\gamma$ in direction $e_{i}-e_{j}$ direction, $\tilde{T}^{\prime}\left(\gamma \mid e_{i}-e_{j}\right)$
} 


\subsubsection{Additive Separability}

The proof of additive separability is staged and inter-leaved with the proof of differentiability. While we cannot in the text convey the full flavor of the additivity and differentiability results, it may be helpful to point out several key insights.

The first observation is that the Lagrangian Lemma implies that there is a common hyperplane tangent to each of the net utility functions at each chosen posterior. This links directional derivatives of the net utility function at distinct optimal posteriors (Lemma 5.11).

Equalization of Directional Derivatives: Suppose $C \in \mathcal{C}$ has a UPS representation $K$, and consider $(\mu, A) \in \mathcal{D}$ and $P \in C(\mu, A)$ with $a, b \in \mathcal{A}(P)$ with $\left\{\bar{\gamma}_{P}^{a}, \bar{\gamma}_{P}^{b}\right\} \subset \tilde{\Gamma}$. Suppose that $\operatorname{both} \tilde{T}_{(j i)}^{a}\left(\bar{\gamma}_{P}^{a}\right)=\tilde{T}_{(j i)}^{b}\left(\bar{\gamma}_{P}^{b}\right)$, then

$$
N_{(j i)}^{a}\left(\bar{\gamma}_{P}^{a}\right)=N_{(j i)}^{b}\left(\bar{\gamma}_{P}^{b}\right)
$$

A second observation is that IUC places structure on the sets of posteriors that can be linked by considering decision problems with equivalent states. In Figure 7, we illustrate this implication of IUC with three states, but the intuition applies generally. Consider a decision problem with three states $\left(\omega_{1}, \omega_{2}, \omega_{3}\right)$ and two actions $A=\{a, b\}$, in which states $\omega_{1}$ and $\omega_{2}$ are equivalent. Figure 7 displays the space of potential priors and posteriors. Suppose that $\bar{\mu}_{1}$ is the prior in the basic problem in which all of the combined probability of $\omega_{1}$ and $\omega_{2}$ is assigned to $\omega_{1}$ and $\bar{\mu}_{2}$ is the prior in the case in which $\omega_{2}$ receives all of the weight. Since $\bar{\mu}_{1}\left(\omega_{1}\right)=\bar{\mu}\left(\omega_{2}\right)$ the line segment connecting these two priors is parallel to the segment connecting $(1,0,0)$ and $(0,1,0)$. The line segment connecting $\bar{\mu}_{1}$ and $\bar{\mu}_{2}$ represents the set of potential priors for which,

$$
\mu\left(\omega_{1}\right)+\mu\left(\omega_{2}\right)=\bar{\mu}_{1}\left(\omega_{1}\right)=\bar{\mu}_{2}\left(\omega_{2}\right),
$$

so that $\left(\bar{\mu}_{1}, A\right)$ and $\left(\bar{\mu}_{2}, A\right)$ are basic versions of $(\mu, A)$.

The above shows that, letting $\mu$ to be an arbitrary prior in this set, IUC places restrictions on the relationship between the optimal posteriors for the problems $(\mu, A),\left(\bar{\mu}_{1}, A\right)$ and $\left(\bar{\mu}_{2}, A\right)$ (Lemma 5.12). Consider $\gamma^{a}$. Bayes rule states that $\gamma^{a}(\omega)=P(a \mid \omega) \mu(\omega) / P(a)$. IUC implies that $P(a \mid \omega)$ and $P(a)$ are the same for all $\mu$ on the segment connecting $\bar{\mu}_{1}$ and $\bar{\mu}_{2}$, including $\bar{\mu}_{1}$ and $\bar{\mu}_{2}$ themselves. This implies that as $\mu$ moves from $\bar{\mu}_{1}$ to $\bar{\mu}_{2}, \gamma^{a}$ and $\gamma^{b}$ are always proportionate to $\mu$. It follows that $\gamma^{a}$ and $\gamma^{b}$ lie at the intersection of a line through $\mu$ and $(0,0,1)$, the dashed grey line in the figure, and a line parallel to the segment connecting $(1,0,0)$ and $(0,1,0)$, the solid red and blue lines in the figures. $\bar{\gamma}_{1}^{a}$ and $\bar{\gamma}_{1}^{b}$ in the figure denote the optimal posteriors for $\left(\bar{\mu}_{1}, A\right)$, and $\bar{\gamma}_{2}^{a}$ and $\bar{\gamma}_{2}^{b}$ the optimal posteriors for $\left(\bar{\mu}_{2}, A\right)$. 


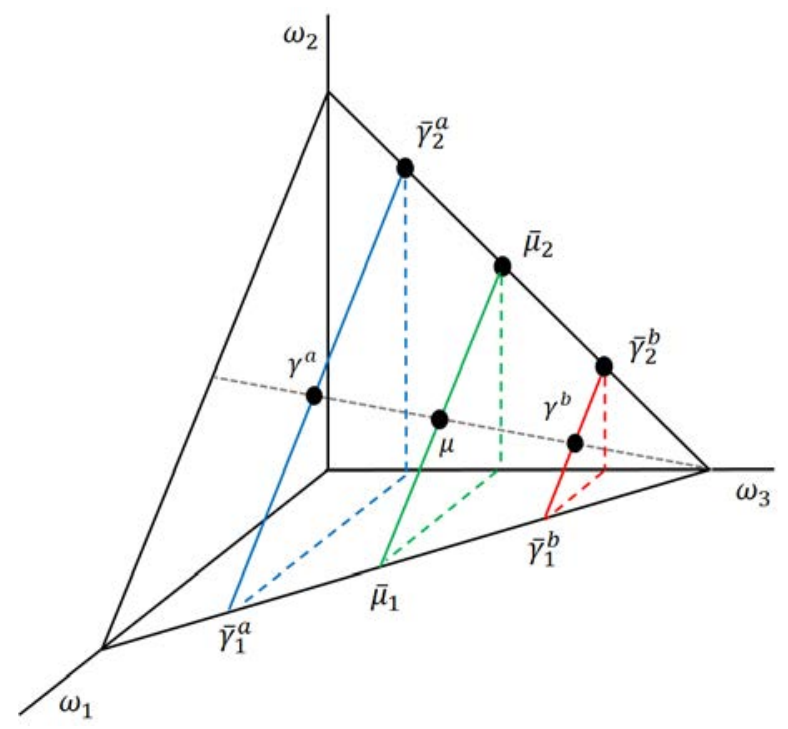

Figure 7: Implications of Compression

These two observations when combined relate the derivatives of $\tilde{T}$ at $\gamma^{a}$ and $\gamma^{b}$ in the Figure. The Lagrangian Lemma implies that there is a hyperplane tangent to both $N\left(\gamma^{a}\right)$ and $N\left(\gamma^{b}\right)$. Suppose that both $\tilde{T}_{(i j)}\left(\gamma^{a}\right)$ and $\tilde{T}_{(i j)}\left(\gamma^{b}\right)$ exist. Since prize-based expected utility is linear, the difference between $\tilde{T}_{(j i)}\left(\gamma^{a}\right)$ and $\tilde{T}_{(j i)}\left(\gamma^{b}\right)$ must equal $u\left(a, \omega_{i}\right)-u\left(a, \omega_{j}\right)-u\left(b, \omega_{i}\right)+u\left(b, \omega_{j}\right)$. Since shifts in $\mu$ from $\bar{\mu}_{1}$ to $\bar{\mu}_{2}$, do not affect prize based utility, $\tilde{T}_{(j i)}\left(\gamma^{a}\right)-\tilde{T}_{(j i)}\left(\gamma^{b}\right)$ must be independent of $\mu$ whenever both derivatives exist (Lemma 5.13).

Consider now two priors $\mu$ and $\hat{\mu}$, each lying between $\bar{\mu}_{1}$ and $\bar{\mu}_{2}$. The four posteriors $\gamma^{a}, \gamma^{b}, \hat{\gamma}^{a}$, and $\hat{\gamma}^{b}$ form a trapezoid in the Figure. If $\tilde{T}$ is differentiable at all four points we would know that $\tilde{T}_{(j i)}\left(\gamma^{a}\right)-\tilde{T}_{(j i)}\left(\gamma^{b}\right)=\tilde{T}_{(j i)}\left(\hat{\gamma}^{a}\right)-\tilde{T}_{(j i)}\left(\hat{\gamma}^{b}\right)$. We show that in general (Lemma 5.17):

$$
\tilde{T}_{\overrightarrow{j i}}\left(\gamma^{a}\right)-\tilde{T}_{\overrightarrow{j i}}\left(\gamma^{b}\right)=\tilde{T}_{\overrightarrow{j i}}\left(\hat{\gamma}^{a}\right)-\tilde{T}_{\overrightarrow{j i}}\left(\hat{\gamma}^{b}\right)
$$

We do so by finding pairs of differentiable points that simultaneously converge to the four posteriors $\gamma^{a}, \gamma^{b}, \hat{\gamma}^{a}$, and $\hat{\gamma}^{b}$.

Equation (11) is close to the rectangle condition for additive separability. To apply the rectangle condition, we deform the simplex so that the trapezoid becomes a rectangle, and then return to the simplex. This results in the following characterization of the directional derivative which we state in terms of the dimension $J$ since it requires $J \geq 4$ (Lemma 5.21):

$$
\tilde{T}_{\overrightarrow{j i}}(\gamma)=\boldsymbol{A}\left(\frac{\gamma(1)}{\gamma(1)+\gamma(J)}\right)+\boldsymbol{B}(\gamma(2), \ldots, \gamma(J-1)),
$$

for some $\boldsymbol{A}: \mathbb{R}_{+} \longrightarrow \mathbb{R}$ and $\boldsymbol{B}: \mathbb{R}^{J-2} \longrightarrow \mathbb{R}$, and all $2 \leq i \neq j \leq J-1$. As (11) must hold for a range of $\gamma(1)$ and $\gamma(J)$ we can show that $\boldsymbol{A}\left(\frac{\gamma(1)}{\gamma(1)+\gamma(J)}\right)$ must be constant (Lemma 5.22). Symmetry then implies that, if $\tilde{T}_{\overrightarrow{j i}}(\gamma)$ does not depend on $\gamma(1)$ and $\gamma(J), \boldsymbol{B}$ cannot depend on any $\gamma(k)$ other than 
$\gamma(i)$ and $\gamma(j)$ (Lemma 5.24). Finally, we use the fact that $\tilde{T}_{(j i)}(\gamma)=\tilde{T}_{(k i)}(\gamma)-\tilde{T}_{(k j)}(\gamma)$ whenever the latter are well defined to establish that there exists a function $f$ on $(0,1)$ such that for all $\gamma \in \tilde{\Gamma}$ (Lemma 5.29):

$$
\tilde{T}_{\overrightarrow{j i}}(\gamma)=f(\gamma(i))-f(\gamma(j))
$$

This function is then used to show that the two-sided directional derivatives $\tilde{T}_{(j i)}$ exist everywhere (Lemma 5.36).

\subsubsection{The Second Order PDE and Shannon Entropy}

Consider again the problem in Figure 7. The Lagrangian Lemma implies that as we shift $\mu$ between $\bar{\mu}_{1}$ and $\bar{\mu}_{2}$, the resulting revealed posteriors satisfy $N_{(j i)}^{a}\left(\gamma^{a}(\mu)\right)=N_{(j i)}^{b}\left(\gamma^{b}(\mu)\right)$. Setting $\mu(t)=$ $t \bar{\mu}_{2}+(1-t) \bar{\mu}_{1}$, we can define $\gamma^{a}(t)=\gamma^{a}(\mu(t))$ and $\gamma^{b}(t)=\gamma^{b}(\mu(t))$ to be the revealed posteriors associated with $\mu(t)$. Given the twice differentiability of $\tilde{T}$, we have $\frac{d}{d t} N_{(j i)}^{a}\left(\gamma^{a}(t)\right)=\frac{d}{d t} N_{(j i)}^{b}\left(\gamma^{b}(t)\right)$, and, since $\omega_{1}$ and $\omega_{2}$ are redundant prized-based utility does not depend on $t$, so that

$$
\frac{d}{d t} \tilde{T}_{(j i)}\left(\gamma^{a}(t)\right)=\frac{d}{d t} \tilde{T}_{(j i)}\left(\gamma^{b}(t)\right)
$$

Finally, note that since $\gamma^{a}(t), \mu(t)$, and $\gamma^{b}(t)$ all lie along a line through $(0,0,1)$, a change in $t$ alters $\gamma^{a}$ proportionately more than $\gamma^{b}$. It follows that

$$
\gamma^{a}(1) \tilde{T}_{(j i)(12)}\left(\gamma^{a}\right)=\gamma^{b}(1) \tilde{T}_{(j i)(12)}\left(\gamma^{b}\right)
$$

Since this equation holds for all $\gamma(1)$, both sides must equal some constant $\kappa^{J}$, and, since $\tilde{T}_{(j i)}(\gamma)=$ $f(\gamma(i))-f(\gamma(j))$, we arrive at

$$
\gamma(1) f^{\prime}(\gamma(1))=\kappa^{J}
$$

A particular solution to this equation is $\kappa^{J} \ln x$. Integrating once more yields the Shannon form:

$$
\tilde{T}=\kappa^{J} \sum_{j} \gamma(j) \ln (\gamma(j))
$$

Other solutions to these differential equations can be rejected as either irrelevant (they sum to a constant because the $\gamma(j)$ sum to a constant), inconsistent with the dependence of $\tilde{T}_{(j i)}$ on solely on $\gamma(i)$ and $\gamma(j)$, or inconsistent with symmetry.

\subsubsection{IUC and Universal Domain}

The proof at this stage has three gaps. First, it applies only to interior posteriors. Second, there is no tie between dimensions $J \geq 4$. Third it does not cover lower dimensional cases. We show next that IUC solves all of these.

The first key observation is that, given $J \geq 4$, all optimal strategies are precisely as if $\kappa^{J}$ applied to all posteriors $\gamma \in \Gamma$ with $|\Omega(\gamma)|=L \leq J$. Note that, as a convex function, the costs are at least as high as the limit of the costs on the boundary. This limit function is in fact the classical 
Shannon entropy function,

$$
T(\gamma) \geq \kappa^{J} \sum_{l=1}^{L} \gamma(l) \ln \gamma(l) .
$$

Even if costs take this minimum value, the known necessary and sufficient conditions for optimality imply that no prior possible states are ever ruled out in an optimal strategies. Hence the behavioral data is precisely as it would be if this function applied to all posteriors, even those that set some prior possible states as impossible.

The final part of the proof uses IUC to iterate down in dimension. To be precise, define $K^{J}$ to be the Shannon cost function with parameter $\kappa^{J}$ for $J \geq 4$ as defined on all posteriors with that state space or below,

$$
K^{J}(\gamma) \equiv \kappa^{J} \sum_{j \in \Omega(\gamma)} \gamma(j) \ln \gamma(j), \text { all } \gamma \in \Gamma \text { with }|\Omega(\gamma)| \leq J
$$

The precise result we establish is that, given any decision problem $(\mu, A) \in \mathcal{D}$ with a prior of cardinality one lower, $|\Omega(\mu)|=J-1$,

$$
P \in C(\mu, A) \text { iff } \exists \lambda \in \hat{\Lambda}\left(\mu, A \mid K^{J}\right) \text { such that } \mathbf{P}_{\lambda}=P .
$$

Note that establishing this completes the proof of the theorem, since it directly implies that $\kappa^{J}=$

$\kappa^{J-1}$ for $J \geq 4$, where the Shannon form was already established, and that the Shannon form and the corresponding parameter apply also to $J=3$, then iteratively to $J=2$, completing the logic.

\section{Existence and Recoverability}

As indicated in the introduction, our remaining results establish necessary and sufficient conditions for a UPS representation. In this section we cover the first stage of this three stage process, by introducing conditions that establish recoverability of the cost function.

\subsection{NIAS, NIAC, and Completeness}

Our general recoverability result rests on three axioms, all of which are necessary for a PS representation of any kind, and indeed apply even more generally. Our first two axioms are required for existence of any CIR. "No Improving Action Switches" (NIAS), due to Caplin and Martin [2015], is based on utility being maximized at each posterior. It insists that all actions chosen maximize expected utility at the corresponding posterior. "No Improving Attention Cycles" (NIAC), adapted from Caplin and Dean [2015], rules out switching attention strategies across problems in a manner that increases overall utility. It insists that attention strategies cannot be shuffled between decision problems in such a manner as to raise total utility across these decision problems.

Axiom A2 No Improving Action Switches (NIAS): Given $(\mu, A) \in D$ and $P \in C(\mu, A)$,

$$
a \in \mathcal{A}(P) \Longrightarrow \bar{u}\left(\bar{\gamma}_{P}^{a}, a\right)=\hat{u}\left(\bar{\gamma}_{P}^{a}, A\right)
$$


where,

$$
\hat{u}(\gamma, A) \equiv \max _{a \in A} \bar{u}(\gamma, a) .
$$

Axiom A3 No Improving Attention Cycles (NIAC): Given $\mu \in \Gamma$ and a finite set

$$
\{(A(m), P(m))\}_{1 \leq m \leq M}
$$

with $(\mu, A(m)) \in D, P(m) \in C(\mu, A(m))$, and $(A(1), P(1))=(A(M), P(M))$,

$$
\sum_{m=1}^{M-1} \hat{U}(\mu, A(m), P(m)) \geq \sum_{m=1}^{M-1} \hat{U}(\mu, A(m), P(m+1)),
$$

where,

$$
\hat{U}(\mu, A, P) \equiv \sum_{\gamma \in \Gamma(P)} \mathbf{Q}_{P}(\gamma) \hat{u}(\gamma, A)
$$

Our third axiom insists that almost all posterior distributions satisfying Bayes' rule can be found in the data for some decision problem. The caveat relates to posteriors that entirely rule out some ex ante possible states of the world. As indicated above, this never happens in the Shannon model.

To state this formally, we let $\Gamma^{C}(\mu)$ denote all revealed posteriors ever observed in any decision problem with the given prior, and correspondingly $\mathcal{Q}^{C}(\mu)$ as distributions over posteriors that are observed in the data. We define also $\Gamma^{C}=\cup_{\mu \in \Gamma} \Gamma^{C}(\mu)$.

Axiom A4 Completeness: Given $\mu \in \Gamma$ :

1. $\Gamma^{C}(\mu)$ contains all interior posteriors, $\tilde{\Gamma}(\mu) \subset \Gamma^{C}(\mu)$.

2. $\Gamma^{C}(\mu)$ is a convex set.

3. If $Q \in Q(\mu)$ is such that $\Gamma(Q) \subset \Gamma^{C}(\mu)$ then $Q \in Q^{C}(\mu)$.

\subsection{Recoverability}

The recoverability result rests on A2-A4 alone.

Theorem 2: Given $C \in \mathcal{C}$ satisfying A2-A4, there exists a function $K \in \mathcal{K}$ such that $C(\mu, A) \subset$ $\hat{P}(\mu, A \mid K)$ all $(\mu, A) \in \mathcal{D}$. This function is unique on $(\mu, Q) \in \mathcal{F}$ with $Q \in Q^{C}(\mu)$.

The proof has two key steps. The first establishes existence of a cost function $K \in \mathcal{K}$ such that $C(\mu, A) \subset \hat{P}(\mu, A \mid K)$ all $(\mu, A) \in \mathcal{D}$ based on NIAS and NIAC. This proof is essentially the same as that of Caplin and Martin [2015] and Caplin and Dean [2015]. ${ }^{7}$ The second is a constructive proof that pins this function down deterministically. The second stage is worth sketching out, not only because of its technical importance, but also because it underlies our characterization of PS representations.

\footnotetext{
${ }^{7}$ The richer data also leads us to change proof method, relying in this case on the work of Rochet [1987].
} 
The procedure for constructing the cost function involves application of the fundamental theorem of calculus. Given $\mu \in \Gamma$ and $\bar{Q} \in \mathcal{Q}^{C}(\mu)$, we first enumerate the possible posteriors $\bar{\gamma}^{n} \in \Gamma(\bar{Q})$ for $1 \leq n \leq N=|\Gamma(\bar{Q})|$ and define corresponding fixed probability weights $\bar{Q}^{n} \equiv \bar{Q}\left(\bar{\gamma}^{n}\right)$. We then construct a path from the prior to the set of posteriors by defining for each $n$ a line

$$
\bar{\gamma}_{t}^{n}=t \bar{\gamma}^{n}+(1-t) \mu
$$

so that at $t=0$ we have $\bar{\gamma}_{0}^{n}=\mu$ and at $t=1$ we have $\bar{\gamma}_{1}^{n}=\bar{\gamma}^{n}$. For each $t$ we consider the distribution $\bar{Q}_{t}$ in which each $\bar{\gamma}_{t}^{n}$ is selected with the same probability as $\bar{\gamma}^{n}$,

$$
\bar{Q}_{t}\left(\bar{\gamma}_{t}^{n}\right)=\bar{Q}^{n}
$$

Note that this construction ensures that the weighted average of the posteriors always averages back to the prior,

$$
\sum_{n} \bar{Q}_{t}\left(\bar{\gamma}_{t}^{n}\right) \bar{\gamma}_{t}^{n}=\sum_{n} \bar{Q}^{n}\left[t \bar{\gamma}^{n}+(1-t) \mu\right]=\mu
$$

so that $\bar{Q}_{t} \in \mathcal{Q}(\mu)$.

Since $\bar{Q}_{t} \in \mathcal{Q}(\mu)$, A4 implies that $\bar{Q}_{t} \in \mathcal{Q}^{C}(\mu)$. Hence for every $t \in[0,1]$, there exists a decision problem $\left(\mu, \bar{A}_{t}\right) \in \mathcal{D}$ and observed data $\bar{P}_{t} \in C\left(\mu, \bar{A}_{t}\right)$ that give rise to the corresponding distribution of revealed posteriors $\mathbf{Q}_{\bar{P}_{t}}=\bar{Q}_{t}$.

Given any cost function $K \in \mathcal{K}$ such that $C(\mu, A) \subset \hat{P}(\mu, A \mid K)$ all $(\mu, A) \in \mathcal{D}$, we then show that,

$$
K\left(\mu, \bar{Q}_{t}\right) \equiv \bar{K}(t)
$$

is convex and continuous in $t \in[0,1]$, and hence almost everywhere differentiable in $t$ with,

$$
\bar{K}(t)=\int_{0}^{1} \bar{K}^{\prime}(t) d t
$$

where the integration is over points of differentiability.

Next we characterize $\bar{K}^{\prime}(t)$. At any point $t$ at which $\bar{K}(t)$ is differentiable, we consider the decision problem $\left(\mu, \bar{A}_{t}\right)$ for which $\bar{Q}_{t}$ is globally, hence locally, optimal. Thinking of shifting locally to a different posterior distribution $Q_{s}$ for $s \in(t-\epsilon, t+\epsilon)$ leads to a first-order condition,

$$
\bar{K}^{\prime}(t)=\sum_{n} \bar{Q}^{n}\left(\left[\bar{\gamma}^{n}-\mu\right] \cdot u\left(\bar{a}_{t}^{n}\right)\right)
$$

where $\bar{a}_{t}^{n}$ is any chosen action associated with $\gamma_{t}^{n} \in \Gamma\left(Q_{t}\right)$ and where the dot product $\left[\bar{\gamma}^{n}-\mu\right] \cdot u\left(\bar{a}_{t}^{n}\right)$ is defined by,

$$
\left[\bar{\gamma}^{n}-\mu\right] \cdot u\left(\bar{a}_{t}^{n}\right) \equiv \sum_{\omega \in \Omega(\mu)}\left[\bar{\gamma}^{n}(\omega)-\mu(\omega)\right] u\left(\bar{a}_{t}^{n}, \omega\right)
$$

Substituting (16) into (15) yields,

$$
K(\mu, \bar{Q})=\sum_{n} \bar{Q}^{n}\left[\bar{\gamma}^{n}-\mu\right] \cdot \int_{0}^{1} u\left(\bar{a}_{t}^{n}\right) d t .
$$


Note that, given $(\mu, \bar{Q}) \in \mathcal{F}$ with $\bar{Q} \in \mathcal{Q}^{C}(\mu)$, enumerating the support $\Gamma(\bar{Q})=\left\{\bar{\gamma}^{n} \mid 1 \leq n \leq N\right\}$ and using the notation above, this cost function is of the form,

$$
K(\mu, \bar{Q}) \equiv \sum_{n} \bar{Q}\left(\bar{\gamma}^{n}\right) T_{\mu}^{C}\left(\bar{\gamma}^{n}, \bar{Q}\right)-T_{\mu}^{C}(\mu, \bar{Q}),
$$

where $T_{\mu}^{C}(\mu, \bar{Q})=0$ and,

$$
T_{\mu}^{C}\left(\bar{\gamma}^{n}, \bar{Q}\right) \equiv\left[\bar{\gamma}^{n}-\mu\right] \cdot \int_{0}^{1} u\left(\bar{a}_{t}^{n}\right) d t
$$

There are three noteworthy aspects of the result. First, the variational logic reflects the economic intuition that marginal utility of improved information should align with its marginal cost. If a large change in payoffs is required to induce a small change in the optimal posterior, learning is costly on the margin. The second point is that many action sets produce the same distribution of posteriors. For example one could shift up all payoffs by a constant amount. What we know is that (17) must be invariant to the particular action set that generates this posterior distribution. In the particular case of adding a constant to all payoffs, invariance follows because state by state differences between prior and posterior average to zero. What the general result tells us is that the corresponding invariance is fully general once A2 through A4 are assumed.

The third point of interest is that the cost function recovered in this general case has much in common with PS cost functions. The key distinction is that $T_{\mu}^{C}\left(\bar{\gamma}^{n}, \bar{Q}\right)$ depends not only on the particular posterior $\bar{\gamma}^{n}$ but also the full distribution of posteriors $\bar{Q}$. Hence the computation for a fixed posterior can be entirely different should the distribution of posteriors change. This differentiates it from the PS form, to which we now turn.

\section{$7 \quad$ PS and UPS Representations}

In this section we introduce axioms for PS and UPS representations.

\subsection{Separability}

As indicated above, the first key step in the PS proof is to rule out dependence of $T_{\mu}^{C}\left(\gamma^{n}, \bar{Q}\right)$ in (17) on the distribution of posteriors. Given $\gamma \in \Gamma(\bar{Q}) \cap \Gamma\left(\bar{Q}^{\prime}\right)$, we want to ensure that,

$$
T_{\mu}^{C}(\gamma, \bar{Q})=T_{\mu}^{C}\left(\gamma, \bar{Q}^{\prime}\right)
$$

This requires an invariance axiom concerning data with shared revealed posteriors. We must be able to find decision problems that produce both distributions using common actions at shared posteriors. The logic of this axiom is demonstrated in Figure 8. Consider again the decision problem $(\mu,\{a, b\})$ of Figure 4. The optimal strategy for this decision problem involves the use of posteriors $\hat{\gamma}^{a}$ and $\hat{\gamma}^{b}$ and so these posteriors would be revealed in the data. Our separability axiom demands that for any arbitrary posterior $\hat{\gamma}^{c}$, such that $\left\{\hat{\gamma}^{b}, \hat{\gamma}^{c}\right\}$ can be the support for an attention strategy feasible from $\mu$, there must exist a corresponding action $c$ such that this pair of posteriors are revealed in the SDSC data from $(\mu,\{b, c\})$, with $\hat{\gamma}^{b}$ still the revealed posterior for action $b$ (see Figure 8a). 


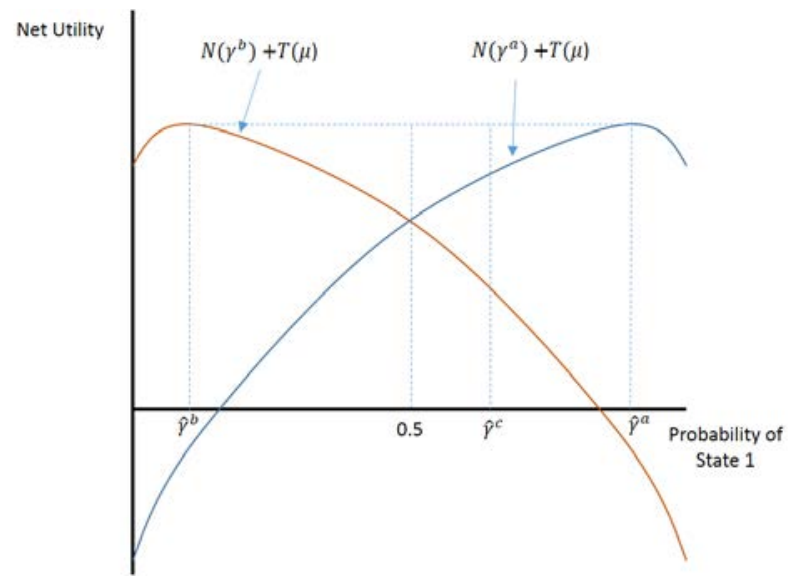

Figure $8 \mathrm{a}$

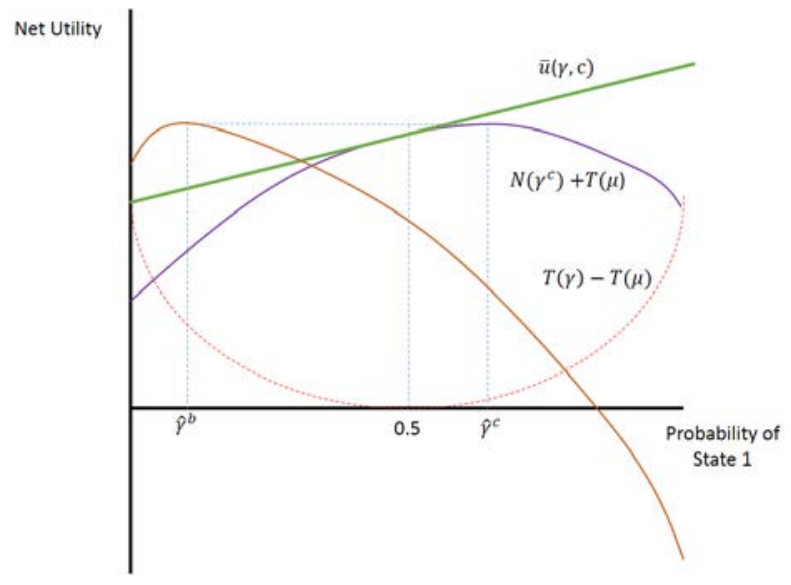

Figure $8 \mathrm{~b}$

The necessity of this axiom for our model is illustrated in Figure 8b. We begin with the hyperplane which defines the optimal strategy in problem $(\mu,\{a, b\})$ which is tangent to the net utility function for action $a$ at $\hat{\gamma}^{a}$ and action $b$ at $\hat{\gamma}^{b}$. Given the ability to shift and tilt the gross utility line defined by the payoffs, it is always possible to find an action $c$ such that the resulting gross utility function, when combined with the cost curve, gives a net utility function which is tangent to the hyperplane precisely at $\hat{\gamma}^{c}$. The Lagrangian Lemma then tell us that $\left\{\hat{\gamma}^{b}, \hat{\gamma}^{c}\right\}$ define the support of an optimal strategy in the resulting decision problem, and so must be observed in the data for $(\mu,\{b, c\})$ as required.

This logic holds more generally, as stated in the following axiom.

Axiom A5 Separability: Given $(\mu, A(1)) \in D, P(1) \in C(\mu, A(1))$, and $Q_{2} \in Q^{C}(\mu)$ with $\Gamma\left(Q_{P(1)}\right) \cap \Gamma\left(Q_{2}\right) \neq \emptyset$, there exists $A(2) \subset A$ and $P(2) \in C(\mu, A(2))$ satisfying $Q_{P(2)}=Q_{2}$ such that $q_{P(1)}(a \mid \gamma)=q_{P(2)}(a \mid \gamma)$ all $\gamma \in \Gamma\left(Q_{P(1)}\right) \cap \Gamma\left(Q_{2}\right)$.

The proof that Separability implies existence of function $T_{\mu}$ such that $T_{\mu}(\gamma)=T_{\mu}^{C}(\gamma, Q)$ in equation (17) for all $Q \in \mathcal{Q}^{C}(\mu)$ is straight forward. It involves standard linear algebra arguments as well as our knowledge of the specific structure of the cost function for each fixed posterior distribution as defined by (17).

While the Separability axiom uses the existential qualifier, in the case of Shannon representations one can specify the precise change in actions needed to generate specified changes in the posteriors. This follows from the invariant likelihood ratio property specified in equation (10). This ratio is enough to pin down the action required in $A(2)$ to generate any $\gamma \in \Gamma\left(Q_{2}\right) / \Gamma\left(\mathbf{Q}_{P(1)}\right)$ using the posteriors in $\Gamma\left(\mathbf{Q}_{P(1)}\right) \cap \Gamma\left(Q_{2}\right)$ and their associated actions. 


\subsection{Convexity Properties}

With Separability, we have a rationalizing cost function of the PS form, but without the required strict convexity. In the next stage of the proof we show that there is no loss of generality in assuming the function to be weakly convex. In terms of rationality, there is no advantage to deliberately throwing away information, so that, even if they were present, concave portions of the cost function would never be acted on. This aspect of the proof is very much analogous to the result of Afriat [1967] that concavity can be assumed of any utility function recovered from optimizing choice in a linear budget set.

While weak convexity is guaranteed, one cannot guarantee strict convexity without additional assumptions. To this end we introduce a non-linearity axiom which insists that if one revealed posterior is a mixture of two others, then the expected utilities cannot be correspondingly mixed. This directly permits the further step from weak to strict convexity.

Axiom A6 Non-linearity: Given $(\mu, A) \in \mathcal{D}, P \in C(\mu, A)$, and distinct $a_{1}, a_{2}, a_{3} \in \mathcal{A}(P)$ with $\bar{\gamma}_{P}^{a_{1}} \neq \bar{\gamma}_{P}^{a_{3}}$

$$
\bar{\gamma}_{P}^{a_{2}}=\alpha \bar{\gamma}_{P}^{a_{1}}+(1-\alpha) \bar{\gamma}_{P}^{a_{3}} \Longrightarrow \bar{u}\left(\bar{\gamma}_{P}^{a_{2}}, a_{2}\right) \neq \alpha \bar{u}\left(\bar{\gamma}_{P}^{a_{1}}, a_{2}\right)+(1-\alpha) \bar{u}\left(\bar{\gamma}_{P}^{a_{3}}, a_{3}\right) .
$$

\subsection{From Some to All Optima}

With axioms A2 through A6, we are able to identify a PS cost function $K \in \mathcal{K}^{P S}$ that rationalizes all observed data, so that $C(\mu, A) \subset \hat{P}(\mu, A \mid K)$. Two additional axioms are required to establish that all optimal strategies are seen, $C(\mu, A)=\hat{P}(\mu, A \mid K)$. We first impose a convexity property on the data.

Axiom A7 Convexity: Given $(\mu, A) \in D, P_{l} \in C(\mu, A)$ for $1 \leq l \leq L$, and probability weights $\alpha(l)>0, P_{\alpha} \in C(\mu, A)$, where,

$$
P_{\alpha}(a \mid \omega) \equiv \sum_{l=1}^{L} \alpha(l) P_{l}(a \mid \omega)
$$

With this, we first show that an arbitrary optimal strategy can be decomposed (using an appropriate mixture operation) into a set of such strategies $\lambda(l)$ with linearly independent posteriors,

$$
\lambda=\sum_{l=1}^{L} \alpha(l) \lambda(l)
$$

Caratheodory's theorem plays the key role in this part of the proof. We then show that this mixture operation correspondingly mixes the data, so that if each of the data sets $\mathbf{P}_{\lambda(l)}$ is observed, Convexity implies that $\mathbf{P}_{\lambda}=\sum_{l=1}^{L} \alpha(l) \mathbf{P}_{\lambda(l)}$ must also be observed.

Our final axiom provides conditions ensuring that each data set $\mathbf{P}_{\lambda(l)}$ with linearly independent posteriors is indeed observed. A key observation in this stage concerns uniqueness of optimal 
strategies. A uniqueness lemma ensures that any optimal strategy that uses linearly independent posteriors is uniquely optimal provided all available actions are chosen. To apply this to strategy $\lambda(l)$, we diminish the payoffs to all actions that are unchosen in this strategy by an arbitrarily small amount. This marginal change ensures that the uniqueness result applies to all correspondingly perturbed decision problems, for each of which $\lambda(l)$ is therefore uniquely optimal. Uniqueness of optimal strategies in a PS representation implies that the corresponding SDSC data is observed.

Our process of taking perturbations allows us to construct for each $\lambda(l)$ a corresponding sequence of action sets that converges to $A$ in the limit in such a way that $\lambda(l)$ is uniquely optimal, hence observed in the data all the way to the limit. To use convergence of this sequence of decision problems to make a conclusion on the limit problem itself requires a continuity axiom. Given $\mu \in \Gamma$ we define a payoff-based metric on the space of actions,

$$
d\left(a, a^{\prime}\right)=\left(\sum_{\omega \in \Omega(\mu)}\left(u(a, \omega)-u\left(a^{\prime}, \omega\right)\right)^{2}\right)^{\frac{1}{2}}
$$

Axiom A8 Continuity: Consider $I \geq 1$ sequences of actions $a^{i}(m)$ with $\lim _{m \rightarrow \infty} a^{i}(m)=\bar{a}^{i}$ for $1 \leq i \leq I$, and define $A(m)=\cup_{i=1}^{I} a^{i}(m)$ and $\bar{A}=\cup_{i=1}^{I} \bar{a}^{i}$. Then given $\mu \in \Gamma$ and $P \in \cap_{m=1}^{\infty} C(\mu, A(m))$,

$$
\mathcal{A}(P) \subset \bar{A} \Longrightarrow P \in C(\mu, \bar{A}) .
$$

This is a very weak condition concerning sequences of choice sets which converge pointwise and which have a subset of actions which remain fixed. If, at every step in the sequence, the same choice behavior is observed (which must therefore only involve choice amongst actions available in all choice sets), then that behavior must also be observed in the limit. In light of our perturbation method, this suffices to establish that all data sets $\mathbf{P}_{\lambda(l)}$ are observed in the original choice set. To complete the proof, we apply the convexity result to show that the data $\mathbf{P}_{\lambda}$ generated by the original optimal strategy is also observed.

\subsection{Existence and Simple Recovery}

We summarize this discussion in the following theorem.

Theorem 3: Data set $C \in \mathcal{C}$ has a PS representation if and only if it satisfies Axioms A2 through A8.

Given a PS cost function, we show that there is a relatively simple way to recover it. Given $\mu \in \Gamma$ and non-degenerate $\bar{Q} \in \mathcal{Q}^{C}(\mu)$, Corollary 2 establishes existence of a choice set $\bar{A}$ such that an inattentive strategy $\eta \in \Lambda^{I}(\mu, \bar{A})$ and a strategy $\lambda=\left(Q_{\lambda}, q_{\lambda}\right) \in \Lambda(\mu, \bar{A})$ with $Q_{\lambda}(\gamma)=\bar{Q}(\gamma)$ are both optimal, hence have equal expected utility net of attention costs,

$$
U(\lambda)-U(\eta)=K(\mu, \bar{Q})-K(\mu, \eta)
$$

By construction, the inattentive strategy is free, $K(\mu, \eta)=0$, so that indifference implies that $K(\mu, \bar{Q})$ is directly computable as the difference in expected utility,

$$
K(\mu, \bar{Q})=U(\lambda)-U(\eta) .
$$




\subsection{LIP and UPS Theorem}

A single invariance axiom takes us from a PS to a UPS representation. Locally Invariant Posteriors (LIP) conveys the idea that, given $P \in C(\mu, A)$, the resulting action-posterior pairs are invariant to various changes in $\mu$ and $A$.

First, if the prior $\mu$ changes to $\mu^{\prime}$ such the posteriors revealed in $(\mu, A)$ are still feasible, then they must still be observed in $C\left(\mu^{\prime}, A\right)$. The necessity of this condition is illustrated in Figure 9 , which again builds on the decision problem $(\mu,\{a, b\})$ with $\mu=0.5$ and optimal posteriors $\hat{\gamma}^{a}$ and $\hat{\gamma}^{b}$. Recall that these posteriors are identified as supporting the highest chord above the prior $\mu$. Consider the prior $\mu^{\prime}$ with $\mu^{\prime}\left(\omega_{1}\right)=0.3$, and note that precisely the same posteriors support the highest chord above this new prior as well, implying that they remain optimal and so must be observed for decision problem $\left(\mu^{\prime},\{a, b\}\right)$.

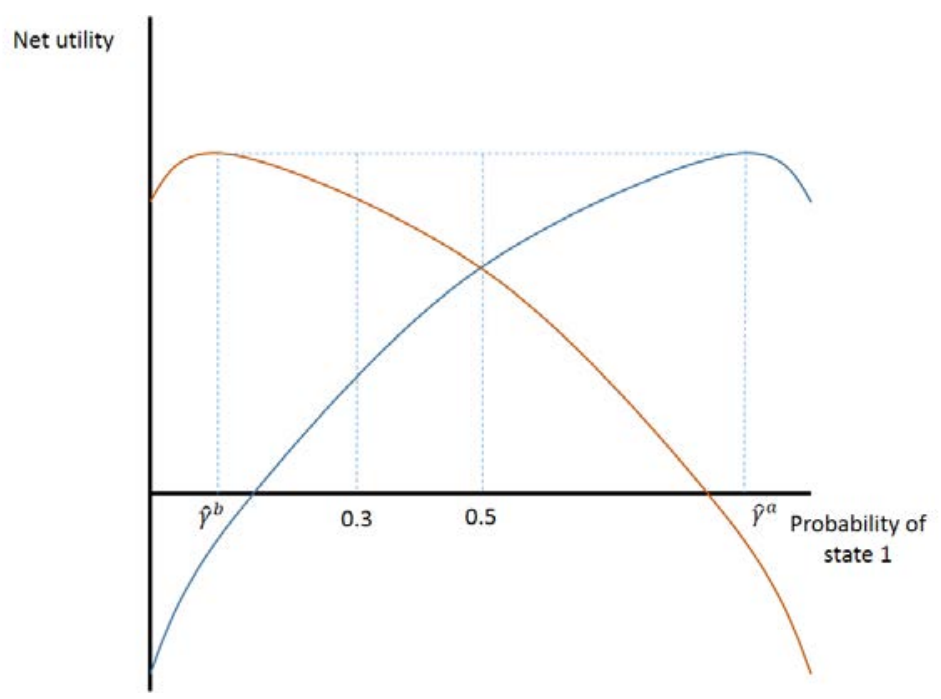

Figure 9: Locally Invariant Posteriors

LIP also requires that, given $P \in C(\mu, A)$, if a new decision problem is defined by deleting some available actions, then the remaining action-posterior pairs must be observed provided Bayesian consistency is retained.

The following formal definition captures both of these invariance properties.

Axiom A9 Locally Invariant Posteriors (LIP): Consider $(\mu, A) \in D, P \in C(\mu, A)$, and probabilities $\rho(a)>0$ on $A^{\prime} \subset A(P)$ with $\sum_{a \in A^{\prime}} \rho(a)=1$. Define $P^{\prime} \in P$ by $A\left(P^{\prime}\right)=A^{\prime}$, $Q_{P^{\prime}}(\gamma)=\sum_{\left\{a \in A^{\prime} \mid \bar{\gamma}_{P}^{a}=\gamma\right\}} \rho(a)$ and:

$$
\mathbf{q}_{P^{\prime}}(a \mid \gamma)=\left\{\begin{array}{c}
\frac{\rho(a)}{\mathbf{Q}_{P}(\gamma)} \text { if } \bar{\gamma}_{P}^{a}=\gamma^{\prime} \\
\mathbf{q}_{P^{\prime}}(a \mid \gamma)=0 \text { else. }
\end{array}\right.
$$


Then $P^{\prime} \in C\left(\sum_{a \in A^{\prime}} \rho(a) \bar{\gamma}_{P}^{a}, A^{\prime}\right)$.

Our fourth theorem shows essentially that a data set with a PS representation has a UPS representation if and only if it satisfies LIP. There is a caveat. For necessity of LIP (Axiom A9), we insist on a link between the posteriors $\Gamma^{C}\left(\mu_{1}\right)$ and $\Gamma^{C}\left(\mu_{2}\right)$ for distinct priors. If prior $\mu_{2}$ lies in the convex hull of posteriors that are revealed posteriors from prior $\mu_{1}$, then these posteriors must be observed from prior $\mu_{2}$ also. We present a simple example in Appendix 4 in which this does not hold. We define regular data sets as those that have this property globally.

Definition 13 Data set $C$ is regular, $C \in \mathcal{C}^{R} \subset \mathcal{C}$, if, given $\mu_{1} \in \Gamma$ and $Q \in \Delta\left(\Gamma\left(\mu_{1}\right)\right)$ with $\Gamma(Q) \subset \Gamma^{C}\left(\mu_{1}\right)$,

$$
\sum_{\gamma \in \Gamma\left(\mu_{2}\right)} \gamma Q(\gamma)=\mu_{2} \Longrightarrow \Gamma(Q) \subset \Gamma^{C}\left(\mu_{2}\right)
$$

Note that the Shannon model generates a data set that is regular, as do other standard entropies. It simplifies our analysis without substantively amending our results to include regularity as a precondition in the necessity proof.

Theorem 4: If $C \in \mathcal{C}$ has a PS representation and satisfies LIP (Axiom A9), it has a UPS representation. If $C \in \mathcal{C}^{R}$ has a UPS representation then it satisfies LIP.

The proof of theorem 4 is lengthy yet conceptually straight forward. It relies on the Lagrangian Lemma and elementary linear algebra. It also relies on invariance of the cost function under affine transforms of the strictly convex function $T_{\mu}$.

Note that between them, theorems 1,3 , and 4 show that data set $C \in \mathcal{C}$ has a Shannon representation if and only if it satisfies Axioms A1 through A9. For the sake of completeness, we establish this as Corollary 3 in Appendix 5.

\section{Further Results}

In this section we provide further results that expand on various features of our representation. We first show how to obtain a representation when the IO observes only a single piece of SDSC for each decision problem - i.e. a choice function rather than a choice correspondence. Second, we describe the relationship between our model and the more traditional model of costly information acquisition in which the DM chooses between information structures consisting of signals, rather than probability distributions over posteriors. Finally we introduce Tsallis entropy (Tsallis [1988]), an alternative formulation to that of Shannon which is of value in describing physical and social systems (see Section 9.3). Costs based on Tsallis entropy fall in the UPS class but do not satisfy IUC, as we demonstrate. 


\subsection{Choice Functions}

To explore the application of our approach to choice functions, we let $\mathcal{C}^{F}$ be the set of data sets in which there is only one observation of SDSC data for each decision problem,

$$
\mathcal{C}^{F} \equiv\left\{C^{F}: \mathcal{D} \rightarrow \mathcal{P} \mid C^{F}(\mu, A) \in \mathcal{P}(\mu, A)\right\}
$$

Given there will be multiple optima in some decision problems, there are several distinct forms of representation that may be of interest. The most obvious approach captures observation of a selection from the optimal choice correspondence.

Definition 14 Data set $C^{F} \in \mathcal{C}^{F}$ has a functional costly information representation (FCIR) $K \in \mathcal{K}$ if, for all $(\mu, A) \in \mathcal{D}$,

$$
C^{F}(\mu, A) \in \hat{P}(\mu, A \mid K) .
$$

It has a FPS/FUPS/F-Shannon representation if it is has an FCIR with $K \in \mathcal{K}^{P S} / \mathcal{K}^{U P S} / K=K_{\kappa}^{S}$ for $\kappa>0$.

We can also consider the case in which the DM mixes among strategies when there are multiple optima, meaning that the observed data falls in the convex hull of the data generated by optimal strategies.

Definition 15 Data set $C^{F} \in \mathcal{C}^{F}$ has a mixed functional costly information representation $(M C I R) K \in \mathcal{K}$ if, for all $(\mu, A) \in \mathcal{D}$,

$$
C^{F}(\mu, A) \in \operatorname{Conv}\{\hat{P}(\mu, A \mid K)\},
$$

It has a MPS/MUPS/M-Shannon representation if it is has an MCIR with $K \in \mathcal{K}^{P S} / \mathcal{K}^{U P S} / K=$ $K_{\kappa}^{S}$ for $\kappa>0$.

Our first observation is that a data set will have a FPS representation if and only if it has an MPS representation. This follows from the fact that, for the PS model, $\hat{P}(\mu, A \mid K)=\operatorname{Conv}\{\hat{P}(\mu, A \mid K)\}$. Thus we can concentrate on identifying conditions which allow for the former type of representation.

The key to functional extensions of our approach is a recoverability result in the spirit of that outlined in Section 6, whereby Axioms A2 through A4 alone are enough to uniquely pin down a rationalizing cost function. In the case of a functional representation, we cannot guarantee that all distributions over posteriors will be observed in the data. However, it is the case that all distributions with linearly independent support will be observed, as all such strategies are uniquely optimal in some decision problem if costs are posterior separable. It is therefore possible to uniquely identify costs for all such attention strategies. Moreover, there is a unique way to extend this cost function to all attention strategies in a manner consistent with posterior separability. If we define mixtures of posterior distributions as in Appendix 2,

$$
Q=\sum_{l=1}^{L} \alpha_{l} Q_{l} \Leftrightarrow Q(\gamma)=\sum_{l=1}^{L} \alpha(l) Q_{l}(\gamma) \text { all } \gamma \in \Gamma(Q)
$$


posterior separability of costs implies that,

$$
\begin{aligned}
Q & =\sum_{l=1}^{L} \alpha_{l} Q_{l} \\
& \Rightarrow K(\mu, Q)=\sum_{l=1}^{L} \alpha_{l} K\left(\mu, Q_{l}\right) .
\end{aligned}
$$

Thus if a data set has a FPS representation then it is possible to uniquely identify those costs $K$ from the data. ${ }^{8}$ Having done so, one can then identify all SDSC which are consistent with optimal behavior with respect to this cost function $\hat{P}(\mu, A \mid K)$. Treating this as a data set, we can then apply the relevant axioms: for an FPS $\hat{P}$ must satisfy Axioms A5-A8, for a FUPS it must also satisfy Axiom A9 and for F-Shannon it must also satisfy Axiom A1. In this way we can construct necessary and sufficient conditions for functional representations.

\subsection{Costly Signal Acquisition}

The standard approach to modeling optimal acquisition of costly information specifies an information structure, consisting of a joint distribution of signals and states. The DM chooses amongst these structures, which are subject to some cost function (see for example Caplin and Dean [2015]). A signal-based strategy comprises an information structure and a mixed action strategy mapping signals to distributions over chosen actions. As is standard, and as we assume in our posteriorbased approach, costs depends only on the information structure, not the action strategy. The DM faced with decision problem $(\mu, A) \in \mathcal{D}$ is modeled as choosing a signal-based strategy to maximize expected utility net of information costs.

The signal-based and posterior-based approaches are equivalent in the sense that a data set can be rationalized by optimal choice of signal-based strategy if and only if it can be rationalized by optimal choice of posterior-based strategies. To go from a CIR in our sense to a corresponding cost function on information structures involves little more than identifying the signals with the posteriors. The argument in the reverse direction involves identifying posteriors associated with the various actions and correspondingly transforming the mixed strategy.

While the data that is characterized is the same using our posterior-based formulation and the standard signal-based formulation, there is a key distinction with regard to testability. Subjective signals are observable only indirectly, through their impact on updating and thereby behavior. From the viewpoint of choice-based analysis, the posterior-based approach has the advantage that it by-passes unobservable signals.

\subsection{Tsallis Entropy and Failures of IUC}

The IUC property seems sufficiently reasonable as to be more widely true. To understand how IUC fails for cost functions other than Shannon, we show how the condition fails for the class of cost functions associated with entropy functions introduced by Tsallis [1988].

\footnotetext{
${ }^{8}$ With regard to attention strategies with linearly dependent support, one can insist that these are only used when optimal according to the recovered cost function.
} 
For $\sigma \in \mathbb{R}, \sigma \neq 1$, the Tsallis entropy of posterior $\gamma \in \Gamma$ is defined by,

$$
T S_{\sigma}(\gamma)=\frac{1}{\sigma-1}\left(1-\sum_{\omega \in \Omega(\gamma)} \gamma(\omega)^{\sigma}\right) \in \mathbb{R}
$$

As $\sigma \rightarrow 1$, Tsallis entropy heads in the limit to Shannon entropy, $H(\gamma)$.

A key property of Tsallis entropy is that it is non-additive. Given two independent probability distributions $\gamma^{1}$ and $\gamma^{2}$, the entropy of the product distribution can be related to the entropy of the marginal distributions,

$$
T S_{\sigma}\left(\gamma^{1} \times \gamma^{2}\right)=T S_{\sigma}\left(\gamma^{1}\right)+T S_{\sigma}\left(\gamma^{2}\right)+(1-\sigma) T S_{\sigma}\left(\gamma^{1}\right) T S_{\sigma}\left(\gamma^{2}\right) .
$$

Shannon entropy $(\sigma=1)$ is the special case of additivity.

Given $\mu \in \Gamma$ it is simple to define the Tsallis cost function for information structures with $\Gamma(Q) \subset \tilde{\Gamma}(\mu)$ in a manner completely analogous to the Shannon model. Costs are related to the expected Tsallis entropy of the posteriors less that of the prior, again with multiplicative factor $\kappa>0$

$$
K_{\kappa}^{T S_{\sigma}}(\mu, Q)=-\kappa\left[\sum Q(\gamma) T S_{\sigma}(\gamma)-T S_{\sigma}(\mu)\right] .
$$

Recall that what is costly is reducing entropy so $K_{\kappa}^{T S_{\sigma}}$ is decreasing in the entropy of the posteriors. $K_{\kappa}^{T S_{\sigma}}(\mu, Q)$ is real-valued for all distributions $Q \in \Delta(\Gamma(\mu)){ }^{9}$

This cost function is a member of the UPS class, and so the resulting behavior satisfies Axioms A2-A9. However it violates IUC. Consider a problem $(\mu, A)$ and suppose that states $\omega_{1}, \omega_{2} \in \Omega(\mu)$ are identical in payoff terms, so that, $u\left(a, \omega_{1}\right)=u\left(a, \omega_{2}\right)$ for all $a \in A$. Consider $P \in C(\mu, A)$ and suppose without loss of generality that each action is chosen from one and only one posterior so that $\mathbf{Q}_{P}\left(\bar{\gamma}_{P}^{a}\right)=P(a)$. Now consider $K_{\kappa}^{T S_{\sigma}}\left(\mu, \mathbf{Q}_{P}\right)$ :

$$
\kappa \sum_{\bar{\gamma}_{P}^{a} \in \Gamma\left(\mathbf{Q}_{P}\right)} \mathbf{Q}_{P}\left(\bar{\gamma}_{P}^{a}\right) \sum_{\omega \in \Omega(\mu)} \bar{\gamma}_{P}^{a}(\omega)\left(\frac{\bar{\gamma}_{P}^{a}(\omega)^{\sigma-1}-1}{\sigma-1}\right)-\kappa \sum_{\omega \in \Omega(\mu)} \mu(\omega)\left(\frac{\mu(\omega)^{\sigma-1}-1}{\sigma-1}\right) ;
$$

where we have pulled out multiplicative factor $\bar{\gamma}_{P}^{a}(\omega)$ to make explicit the relationship to a constant elasticity function. Substituting using Bayes' rule, $\bar{\gamma}_{P}^{a}(\omega)=\frac{P(a \mid \omega) \mu(\omega)}{P(a)}$ and invoking $\sum_{\omega} P(a \mid \omega)=1$,

\footnotetext{
${ }^{9} \mathrm{~A}$ subtle point is that there are cases in which an ex ante possible state may be ruled out, as when $\Omega(\mu)=$ $\left\{\omega_{1}, \omega_{2}, \omega_{3}\right\}$ yet $\gamma \in \Gamma(Q)$ has support $\Omega(\gamma)=\left\{\omega_{1}, \omega_{2}\right\}$. The above formula correctly deals with this case when when $\sigma>0$ because the contribution of these terms to the sum is zero so that their exclusion is immaterial.

Matters are slightly more complex when $\sigma<0$. In this case there are infinite costs to ruling out ex ante possible states. This calls for care in specifying the Tsallis attention cost function. Given $\mu \in \Gamma$, the corresponding cost function is:

$$
K_{\kappa}^{T S_{\sigma}}=\left\{\begin{array}{cc}
\kappa\left[\sum Q(\gamma) T S_{\sigma}(\gamma)-T S_{\sigma}(\mu)\right] & \text { if } \Omega(\gamma)=\Omega(\mu) \text { all } \gamma \in \Gamma(Q) \\
\infty & \text { if } \Omega(\gamma) \neq \Omega(\mu) \text { some } \gamma \in \Gamma(Q)
\end{array}\right.
$$

The need to depart from the standard specification of Tsallis entropy in the above cases is due to what is essentially a missing argument. The standard Tsallis entropy function makes no explicit reference to the prior. Yet the cost of making an ex ante possible state impossible becomes unboundedly high at the margin when $\sigma<0$, so that making it free to entirely rule such a state out would be inappropriate.
} 
leads to the following expression for Tsallis costs in terms of SDSC data:

$$
\begin{aligned}
K_{\kappa}^{T S_{\sigma}}\left(\mu, \mathbf{Q}_{P}\right)= & \sum_{a \in \mathcal{A}(P)} \sum_{\omega \in \Omega(\mu)} P(a \mid \omega) \mu(\omega)^{\sigma}\left[\frac{(P(a \mid \omega) / P(a))^{\sigma-1}-1}{\sigma-1}\right] \\
& -\sum_{\omega \in \Omega(\mu)} \mu(\omega)\left(\frac{\mu(\omega)^{\sigma-1}-1}{\sigma-1}\right)
\end{aligned}
$$

Now suppose that IUC holds so that $P \in C(\mu, A)$ implies $P\left(a \mid \omega_{1}\right)=P\left(a \mid \omega_{2}\right)$ for all $a \in A$. We now focus on the part of this expression associated with a single action $a \in A$ and the two states $\omega_{1}$ and $\omega_{2}$ :

$$
\begin{aligned}
& P\left(a \mid \omega_{1}\right) \mu\left(\omega_{1}\right)^{\sigma} \frac{\left(P\left(a \mid \omega_{1}\right) / P(a)\right)^{\sigma-1}-1}{\sigma-1}+P\left(a \mid \omega_{2}\right) \mu\left(\omega_{2}\right)^{\sigma} \frac{\left(P\left(a \mid \omega_{2}\right) / P(a)\right)^{\sigma-1}-1}{\sigma-1} \\
= & \left(P\left(a \mid \omega_{1}\right) \frac{\left(P\left(a \mid \omega_{1}\right) / P(a)\right)^{\sigma-1}-1}{\sigma-1}\right)\left[\mu\left(\omega_{1}\right)^{\sigma}+\mu\left(\omega_{2}\right)^{\sigma}\right] .
\end{aligned}
$$

We now compare this to the cost that would be incurred if $\omega_{1}$ and $\omega_{2}$ were instead collapsed into the single state $\omega_{1}$ with prior probability $\mu\left(\omega_{1}\right)+\mu\left(\omega_{2}\right)$. If, as specified by IUC, the choice probabilities remain $P\left(a \mid \omega_{1}\right)$

$$
\left(P\left(a \mid \omega_{1}\right) \frac{\left(P\left(a \mid \omega_{1}\right) / P(a)\right)^{\sigma-1}-1}{\sigma-1}\right)\left[\mu\left(\omega_{1}\right)+\mu\left(\omega_{2}\right)\right]^{\sigma} .
$$

If $\sigma<1$ then the decision maker finds it more costly to learn about $\omega_{1}$ and $\omega_{2}$ separately than together,

$$
\mu\left(\omega_{1}\right)^{\sigma}+\mu\left(\omega_{2}\right)^{\sigma}>\left(\mu\left(\omega_{1}\right)+\mu\left(\omega_{2}\right)\right)^{\sigma} .
$$

If $\sigma>1$, the opposite is the case. It is clear that these changes in the marginal cost of information mean that the same $P\left(a \mid \omega_{1}\right)$ cannot generally be optimal in the original problem and its basic form, leading to a violation of IUC.

Only if $\sigma=1$ does the DM treat the two scenarios as equivalent. Recall that as $\sigma \rightarrow 1$, Tsallis entropy approaches Shannon entropy. Shannon entropy is therefore the special case in which the agent is indifferent between aggregating and separating states. This is the essence of the IUC axiom. With Shannon, the cost of implementing $P(a \mid \omega)$ rises proportionately with $\mu(\omega)$, whereas with Tsallis entropy costs rise more than proportionately with $\mu(\omega)$ when $\sigma>1$, and less than proportionately when $\sigma<1$. The implication is that when $\sigma<1$, information is proportionately cheaper in more likely states, so that an agent would appear to pay greater attention in such states.

\section{Relation to the Literature}

\subsection{Existing Characterizations of the Shannon Model}

Several recent papers have provided insights into the behavior implied by the Shannon model. Matejka and McKay [2015] provide a generalized logit formula for optimal SDSC probabilities $P(a \mid \omega)$ in the Shannon model. Caplin and Dean [2013], Stevens [2014], and Caplin et al. [2016] 
show that addition of appropriate complementary slackness conditions renders these necessary and sufficient.

While insightful, such conditions for optimality are not directly revealing of the behavioral patterns that the model produces. In contrast, our analysis is of value in this regard. Indeed, many of the tools we describe - such as the posterior based approach to optimal strategies in the Shannon model and the geometry of net utility functions - have already proved useful in economic research since their introduction in Caplin and Dean [2013] (see for example Caplin et al. [2015] and Martin [2017]). For example, in the UPS case, LIP makes it relatively easy to derive comparative static results as priors change.

A more closely related analysis is that of de Oliveira [2013], who also axiomatizes a form of decision making given a Shannon cost function. The key difference is that de Oliveira [2013] places axioms on preference orderings over menus, whereas we place axioms on choices as revealed in SDSC data. There is therefore no obvious relationship between the axioms. One possible exception is that IUC appears related to de Oliveira [2013]'s independence of orthogonal decision problem (IODP) axiom. IODP involves indifference between solving two decision problems with independent payoffs together or separately. We early on conjectured that we would need both IUC and IODP to generate the Shannon form. We only later realized that IUC alone was sufficient. It is therefore possible that IUC implies IODP. de Oliveira [2013] also does not consider generalizations of the Shannon model.

Pioneering work by Shannon [1948] and Khinchin [1957] provides direct axiomatizations of Shannon entropy. These axiomatizations focus on properties of the measure of information itself, rather than on how basing learning costs on them impacts optimal behavior. Axioms such as continuity, being maximal at uniformity, being invariant to zero probability events, and satisfaction of additivity conditions are shown to imply the Shannon entropy function for probability distributions. This work is therefore focussed on properties of measures of disorder, rather than understanding the behavioral implications of associated attention cost functions.

\subsection{Behavioral Evidence Against the Shannon Model}

The use of the Shannon model is often justified on information theoretic grounds (see for example Sims [2003] and Matejka and McKay [2015]). In particular, mutual information is related to the rate of information flow needed to generate a given conditional distribution of signals given a distribution of states, assuming optimal coding (see for example Cover and Thomas [2012] chapter 10). Yet the experimental literature in economics and psychology establishes that there are important behavioral reasons to look beyond that model.

The most direct evidence derives from experiments that collect SDSC data to which the above characterizations apply. This fledgling literature exhibits behavioral patterns that are inconsistent with the Shannon model. One key problem relates to the elasticity of chosen posteriors to changes in the underlying rewards. The Shannon model makes sharp and precise predictions about the rate at which subjects improve their accuracy in response to improved incentives. A single decision problem is enough to pin down the one parameter in the model and so the "expansion path" of information acquisition in response to changed incentives. Caplin and Dean [2013] show that the improvement in expected utility as the incentive to learn rises is significantly lower than the Shannon model predicts in a simple two state, two action set-up. 
A second behavioral limitation of the Shannon model is that it makes no allowance for perceptual distance. Yet perceptual distance is critical in many every day decisions, as when good decisions require the DM to differentiate between alternative pricing schemes: it seems likely that prices which are closer together will be harder to distinguish than those which are far apart. By way of conceptual confirmation, Dean and Neligh [2017] design an experiment with 100 balls on a screen, of which a random number (between 40 and 60) are red, with the remainder blue. Subjects are tasked with correctly identifying which color ball is in the majority. The Shannon model implies that they must be just as good at the task when there are 51 red balls on the screen as when there are 60 , which is strongly rejected by the data.

An earlier literature in psychology also demonstrates behavior that does not fit with the Shannon model. Woodford [2012] discusses the experimental results of Shaw and Shaw [1977], in which a subject briefly sees a signal which may appear at one of a number of locations on the screen. Their task is to accurately reproduce the location of this briefly seen signal. According to the Shannon cost function, the actual location, being payoff irrelevant, should also be irrelevant to task performance. Yet in practice, performance is superior at locations that occur more frequently.

\subsection{PS Models}

UPS models were introduced in Caplin and Dean [2013]. They are rich enough to allow for many of the behavioral findings that call the Shannon model into question. With regard to incentives, Caplin and Dean [2013] develop a simple two parameter UPS model that generalizes the Shannon model. They find that the additional degree of freedom leads to a significantly better fit of the data according to the Akaike Information Criterion. With regard to perceptual distance, while rejecting the Shannon model, Dean and Neligh [2017] find (weak) support for LIP, and hence the UPS model. Finally, note that while the results of Shaw and Shaw [1977] are inconsistent with the Shannon model, they are consistent with the UPS model. In the Tsallis model with $\sigma<1$, for example, learning about unlikely states is proportionately more expensive than about likely states. This produces a commensurately greater error rate, as in the experiment.

Since their introduction, several papers have made use of UPS costs functions - see for example Gentzkow and Kamenica [2014], Steiner et al. [2015], Clark [2016] and Morris and Strack [2017]. In part this is due to their flexibility in rationalizing behavior. It is also due in part to the fact that UPS models make available familiar Lagrangian methods of optimization.

The current paper is the first to introduce the more general class of PS cost function. As noted, this allows the costs to vary depending on prior beliefs. We expect this additional flexibility also to prove useful in understanding behavior and in economic modeling. Indeed alternative forms of entropy have proven valuable in other disciplines. There are many settings in which the additional flexibility they allow for leads to a better ability to describe physical and social systems. Examples include internet usage (Tellenbach et al. [2009]), machine learning (Maszczyk and Duch [2008]), statistical mechanics (Lenzi et al. [2000]), and many other applications in physics (Beck [2009]). See Gell-Mann and Tsallis [2004] for a review. In these cases, the additivity property of Shannon entropy is found to be unhelpful in describing the phenomena of interest.

Interestingly, the literature in information theory and on the design of experiments has also focussed on PS cost functions. For example, the Blackwell-Sherman-Stein Theorem shows that PS functions can be used to characterize the property of statistical sufficiency, and so provide 
an alternative characterization of Blackwell's theorem. The theorem states that an information structure $\pi$ is statistically sufficient for $\pi^{\prime}$ (i.e. $\pi$ Blackwell dominates $\pi^{\prime}$ ) if and only if,

$$
\sum_{\gamma \in \Gamma\left(Q_{\pi}\right)} Q_{\pi}(\gamma) T(\gamma) \geq \sum_{\gamma \in \Gamma\left(Q_{\pi^{\prime}}\right)} Q_{\pi^{\prime}}(\gamma) T(\gamma)
$$

for every continuous, (weakly) convex $T$, where $Q_{\pi}$ is the distribution over posteriors generated by $\pi$ (see for example Le Cam [1996]). ${ }^{10}$ Torgersen [1991] further shows that the class of PS cost functions can be characterized by properties of the costs themselves. Specifically, the (weakly convex) PS class of cost function of information structures characterizes monotonicity in Blackwell informativeness and linearity in a natural mixture operation.

\subsection{Alternative Models of Limited Attention}

Our work belongs to a recent literature which characterizes the behavior associated with models of incomplete attention - see for example Masatlioglu et al. [2012], Manzini and Mariotti [2014] and Steiner and Stewart [2016]. It is also related to significant bodies of work on costly information acquisition with very different forms of cost function. The most ubiquitous such model is search theoretic, involving a fixed cost of uncovering each available option (e.g. Caplin et al. [2011]). Other approaches include costly purchase of normal signals (Verrecchia [1982], Llosa and Venkateswaran [2012] and Colombo et al. [2014] ) and "all or nothing" information costs (Reis [2006]). Even in the rational inattention literature alternative cost functions have been provided. For example, Paciello and Wiederholt [2014] consider costs that are convex in mutual information, while Sims [2003] considers a model in which there is a hard constraint on the amount of mutual information a DM can use. Inspired by the findings of Shaw and Shaw [1977], Woodford [2012] considers a cost function which is linear in Shannon capacity, rather than Shannon mutual information. Another ongoing body of work to which our modeling relates is the sparsity-based model of Gabaix [2014]. This model is based on a distinct form of attention cost function involving fixed costs of comprehending individual characteristics of options. The question of how these other cost functions restrict behavior, and so how they differ from the PS class, remains open.

\section{Concluding Remarks}

Together our results provide necessary and sufficient conditions for cost functions of increasing specificity. Theorem 3 states that Axioms A2-A8 are necessary and sufficient for the existence of a Posterior Separable attention cost function. In addition, given a Posterior Separable cost function, Theorem 4 states that Locally Invariant Posteriors (Axiom A9) is necessary and sufficient for the existence of a Uniformly Posterior Separable cost function. Finally, given a Uniformly Posterior Separable cost function, Theorem 1 states that Invariance under Compression (Axiom A1) is necessary and sufficient for the cost function to take the Shannon form. In addition, Theorem 2 states that Axioms A1-A3 are sufficient for there to exist a unique attention cost function that represents the data.

\footnotetext{
${ }^{10}$ We thank Daniel Csaba for pointing this out to us.
} 


\section{References}

Sydney N. Afriat. The Construction of Utility Functions from Expenditure Data. International Economic Review, 8(1):67-77, 1967.

Marina Agranov and Pietro Ortoleva. Stochastic choice and preferences for randomization. Available at SSRN $2644784,2015$.

Jose Apesteguia and Miguel A Ballester. Single-crossing random utility models. 2016.

Christian Beck. Generalised information and entropy measures in physics. Contemporary Physics, 50(4):495-510, 2009.

David Blackwell. Comparison of experiments. In Proceedings of the second Berkeley symposium on mathematical statistics and probability, volume 1, pages 93-102, 1951.

Henry David Block and Jacob Marschak. Contributions to Probability and Statistics, volume 2, chapter Random orderings and stochastic theories of responses, pages 97-132. Stanford University Press, 1960.

Andrew Caplin and Mark Dean. Behavioral implications of rational inattention with shannon entropy. NBER Working Papers 19318, National Bureau of Economic Research, Inc, August 2013.

Andrew Caplin and Mark Dean. Revealed preference, rational inattention, and costly information acquisition. The American Economic Review, 105(7):2183-2203, 2015.

Andrew Caplin and Daniel Martin. A testable theory of imperfect perception. The Economic Journal, 125(582):184-202, 2015.

Andrew Caplin, Mark Dean, and Daniel Martin. Search and satisficing. The American Economic Review, 101(7):2899-2922, 2011.

Andrew Caplin, John Leahy, and Filip Matějka. Social learning and selective attention. Technical report, National Bureau of Economic Research, 2015.

Andrew Caplin, Mark Dean, and John Leahy. Rational inattention, optimal consideration sets and stochastic choice. 2016.

Raj Chetty, Adam Looney, and Kory Kroft. Salience and taxation: Theory and evidence. American Economic Review, 99(4):1145-1177, 2009.

Aubrey Clark. Contracts for information acquisition. 2016.

Luca Colombo, Gianluca Femminis, and Alessandro Pavan. Information acquisition and welfare. The Review of Economic Studies, 81:1438-1483, 2014.

Thomas M Cover and Joy A Thomas. Elements of information theory. John Wiley \& Sons, 2012.

Henrique de Oliveira. Axiomatic foundations for entropic costs of attention. Mimeo, Northwestern University, 2013.

Mark Dean and Nathaniel Neligh. Experimental tests of rational inattention. 2017. 
Ambuj Dewan and Nathaniel Neligh. Estimating information cost functions in models of rational inattention. 2017.

Xavier Gabaix. A sparsity-based model of bounded rationality. The Quarterly Journal of Economics, 129(4):1661-1710, 2014.

Murray Gell-Mann and Constantino Tsallis. Nonextensive entropy: interdisciplinary applications. Oxford University Press, 2004.

Matthew Gentzkow and Emir Kamenica. Costly persuasion. The American Economic Review, 104(5):457-462, 2014.

Friedrich August Hayek. Economics and knowledge. Economica, 4(13):33-54, 1937.

Friedrich August Hayek. The use of knowledge in society. The American economic review, pages 519-530, 1945.

Christian Hellwig, Sebastian Kohls, and Laura Veldkamp. Information choice technologies. The American Economic Review, 102(3):35-40, 2012.

Akovlevich Khinchin. Mathematical Foundations of Information Theory, volume 434. Courier Corporation, 1957.

Ian Krajbich and Antonio Rangel. Multialternative drift-diffusion model predicts the relationship between visual fixations and choice in value-based decisions. Proceedings of the National Academy of Sciences, 108(33):13852-13857, 2011.

L Le Cam. Comparison of experiments: A short review. Lecture Notes-Monograph Series, pages 127-138, 1996.

EK Lenzi, RS Mendes, and LR Da Silva. Statistical mechanics based on renyi entropy. Physica A: Statistical Mechanics and its Applications, 280(3):337-345, 2000.

Luis Gonzalo Llosa and Venky Venkateswaran. Efficiency with endogenous information choice. Unpublished working paper. University of California at Los Angeles, New York University, 2012.

R. D. Luce. Individual Choice Behavior: A Theoretical Analysis. New York: Wiley, 1959.

Bartosz Mackowiak and Mirko Wiederholt. Optimal sticky prices under rational inattention. American Economic Review, 99(3):769-803, June 2009.

Paola Manzini and Marco Mariotti. Stochastic choice and consideration sets. Econometrica, 82(3):1153-1176, 2014.

Paola Manzini and Marco Mariotti. Dual random utility maximisation. 2016.

Daniel Martin. Strategic pricing with rational inattention to quality. Mimeo, New York University, 2017.

Yusufcan Masatlioglu, Daisuke Nakajima, and Erkut Y Ozbay. Revealed attention. American Economic Review, 102(5):2183-2205, 2012.

Tomasz Maszczyk and Włodzisław Duch. Comparison of shannon, renyi and tsallis entropy used in decision trees. In International Conference on Artificial Intelligence and Soft Computing, pages 643-651. Springer, 2008. 
Filip Matejka and Alisdair McKay. Rational inattention to discrete choices: A new foundation for the multinomial logit model. American Economic Review, 105(1):272-98, 2015.

Filip Matějka. Rationally inattentive seller: Sales and discrete pricing. The Review of Economic Studies, 83(3):1156-1188, 2015.

Daniel McFadden. Revealed stochastic preference: A synthesis. Economic Theory, 26(2):245-264, 2005.

Jordi Mondria. Portfolio choice, attention allocation, and price comovement. Journal of Economic Theory, 145(5):1837-1864, 2010.

Stephen Morris and Philipp Strack. The wald problem and the equivalence of sequential sampling and static information costs. 2017.

Henrique Oliveira, Tommaso Denti, Maximilian Mihm, and Kemal Ozbek. Rationally inattentive preferences and hidden information costs. Theoretical Economics, 12(2):621-654, 2017.

Luigi Paciello and Mirko Wiederholt. Exogenous information, endogenous information and optimal monetary policy. The Review of Economic Studies, 83:356-388, 2014.

Ricardo Reis. Inattentive producers. Review of Economic Studies, 73(3):793-821, 2006.

Marcel K Richter. Revealed preference theory. Econometrica: Journal of the Econometric Society, pages 635-645, 1966.

Jean-Charles Rochet. A necessary and sufficient condition for rationalizability in a quasi-linear context. Journal of Mathematical Economics, 16(2):191-200, April 1987.

R. Tyrrell Rockafellar. Convex Analysis. Princeton University Press, Princeton, 1970.

C. E. Shannon. A mathematical theory of communication. Bell System Technical Journal, 27(3):379-423, 1948.

M. L. Shaw and P. Shaw. Optimal allocation of cognitive resources to spatial locations. $J$ Exp Psychol Hum Percept Perform, 3(2):201-211, May 1977.

Christopher A. Sims. Stickiness. Carnegie-Rochester Conference Series on Public Policy, 49(1):317356, December 1998.

Christopher A. Sims. Implications of Rational Inattention. Journal of Monetary Economics, 50(3):665-690, 2003.

Jakub Steiner and Colin Stewart. Perceiving prospects properly. American Economic Review, 2016.

Jakub Steiner, Colin Stewart, and Filip Matejka. Rational inattention dynamics: Inertia and delay in decision-making. Centre for Economic Policy Research, 2015.

Luminita Stevens. Coarse pricing policies. Available at SSRN 2544681, 2014.

Bernhard Tellenbach, Martin Burkhart, Didier Sornette, and Thomas Maillart. Beyond shannon: Characterizing internet traffic with generalized entropy metrics. In International Conference on Passive and Active Network Measurement, pages 239-248. Springer, 2009.

Louis L Thurstone. A law of comparative judgment. Psychological review, 34(4):273, 1927. 
Erik Torgersen. Comparison of statistical experiments. Number 36. Cambridge University Press, 1991.

Constantino Tsallis. Possible generalization of boltzmann-gibbs statistics. Journal of statistical physics, 52(1-2):479-487, 1988.

Robert Verrecchia. Information acquisition in a noisy rational expectations economy. Econometrica, 50(6):1415-1430, 1982.

EH Weber. De tactu. Koehler, Leipzig, 1834.

Michael Woodford. Information constrained state dependent pricing. Journal of Monetary Economics, 56(S):S100-S124, 2009.

Michael Woodford. Inattentive valuation and reference-dependent choice. Mimeo, Columbia University, 2012.

Ming Yang. Coordination with flexible information acquisition. Journal of Economic Theory, 158:721-738, 2015. 